\title{
A Random Forest Assisted Evolutionary Algorithm for Data-Driven Constrained Multi-Objective Combinatorial Optimization of Trauma Systems
}

\author{
Handing Wang, Member, IEEE, Yaochu Jin, Fellow, IEEE
}

\begin{abstract}
Many real-world optimization problems can be solved by using the data-driven approach only, simply because no analytic objective functions are available for evaluating candidate solutions. In this work, we address a class of expensive datadriven constrained multi-objective combinatorial optimization problems, where the objectives and constraints can be calculated only on the basis of large amount of data. To solve this class of problems, we propose to use random forests and radial basis function networks as surrogates to approximate both objective and constraint functions. In addition, logistic regression models are introduced to rectify the surrogate-assisted fitness evaluations and a stochastic ranking selection is adopted to further reduce the influences of the approximated constraint functions. Three variants of the proposed algorithm are empirically evaluated on multi-objective knapsack benchmark problems and two realworld trauma system design problems. Experimental results demonstrate that the variant using random forest models as the surrogates are effective and efficient in solving data-driven constrained multi-objective combinatorial optimization problems.
\end{abstract}

Index Terms-Data-driven optimization, constrained multiobjective combinatorial optimization, evolutionary algorithm, surrogate, random forest, radial basis function networks, trauma systems

\section{INTRODUCTION}

Many real-world applications involve solving constrained combinatorial optimization problems whose feasible regions are not convex, seriously limiting the search capability of mathematical programming methods [1]. Therefore, metaheuristics such as evolutionary algorithms (EAs) have recently become popular for handling combinatorial optimization [2], [3]. The use of EAs to solve combinatorial optimization becomes less practical, however, when fitness evaluations of candidate solutions rely on time-consuming numerical simulations or expensive physical experiments rather than computationally cheap analytic functions [4]. Optimization problems that are solved with the help of simulation, experimental or other types

This work was supported in part by an EPSRC grant (No. EP/M017869/1) and in part by the National Natural Science Foundation of China (No. 61590922). (Corresponding author: Yaochu Jin)

H. Wang is with School of Artificial Intelligence, Xidian University, Xi'an 710071 , China. The work was done when she was with the Department of Computer Science, University of Surrey, Guildford, GU2 7XH, UK. (e-mail: hdwang@xidian.edu.cn).

Y. Jin is with the Department of Computer Science, University of Surrey, Guildford, GU2 7XH, U.K. He is also affiliated with the Department of Computer Science and Technology, Taiyuan University of Science and Technology, Taiyuan 030024, China, and the State Key Laboratory of Synthetical Automation for Process Industries, Northeastern University, Shenyang, China. (e-mail: yaochu.jin@surrey.ac.uk). of data are termed data-driven optimization [5]. Typically, surrogates [4] need to be built using the data to substitute the fitness function, in part or completely, in data-driven optimization. According to [5], data-driven surrogate-assisted optimization can be divided into offline and online data-driven, depending on whether a certain amount of new data can be made available during the optimization process. In the last decade, data-driven surrogate-assisted evolutionary algorithms (SAEAs) [6] have found many successful real-world applications, such as aerodynamic design [7], [8], microwave design [9], furnace design [10], and circuit design [11].

Most existing SAEAs have been developed for online datadriven optimization of continuous problems, where appropriate surrogates are chosen and model management strategies are designed to make sure that the EA is able to find the best solution with the given computation budget [12]. A wide range of regression models have been adopted as the surrogates, such as Kriging model (Gaussian processes regression model) [13], [14], radial basis function (RBF) networks [15]-[18], polynomial regression [19], and artificial neural networks [20], [21]. Since different regression models have different strengths and weaknesses, multiple surrogate models are combined as an ensemble in a single SAEA to increase its robustness [22][25].

Model management strategies distinguish themselves mainly in the criteria for selecting new candidate solutions to be evaluated using the expensive objective functions [4]. In generation-based model management strategies, the updating frequency can be fixed [21] or self-adaptive [20]. In individualbased model management strategies, promising and uncertain solutions according to the surrogate model [26]-[28] are evaluated using the expensive objective functions. A large number of SAEAs for single-objective optimization [6], [25], [29], [30], multi-objective optimization [12], [22], [31], [32], and many-objective optimization [13], [33] have been proposed.

By contrast, very few SAEAs have been dedicated to expensive data-driven mixed-integer or combinatorial optimization problems [34]. Typically, regression models widely used for surrogate-assisted continuous optimization are directly adopted as surrogates for mixed-integer optimization problems [35][37] or combinatorial optimization [38]-[40]. Note that many combinatorial optimization problems can be better solved by incorporating domain knowledge, where surrogate models can be helpful [41]-[43].

Likewise, relatively little work has been carried out on surrogate-assisted optimization of constrained problems [44]. 
Constraints become relevant for SAEAs in different situations. For example, the evaluation of the constraint functions may be time-consuming, and consequently, surrogates need to be built for constraints [29], [45], [46]. Since constraints can be handled using penalty functions [47], surrogates are built to approximate the penalty function instead of the individual constraint functions [48], [49]. As whether a candidate solution is feasible or not can be seen as a classification problem, support vector machine [50], [51], k-nearest neighbors algorithm [52], and linear hyper-plane estimator [53] have been employed to distinguish feasible solutions from infeasible ones. Surrogates were also used as a way of manipulating the feasible regions to facilitate the EA to find the global optimum possibly located in an isolated region [54]. Even if the constraint functions are computationally inexpensive, they can still have considerable impact on SAEAs. For example, it has been found in [55] whether the infeasible samples should be used for training the surrogates have significant influences on the search performance of SAEAs.

To summarize, most existing data-driven surrogate-assisted optimization algorithms are developed for solving nonconstrained continuous optimization problems despite the fact that many real-world problems are constrained combinatorial optimization problems. To fill the gap, this work aims to deal with a class of data-driven constrained multi-objective combinatorial optimization problems, where each evaluation of the objectives and constraints involves a large amount of historical data and therefore is time-consuming. Since EAs typically need a large number of fitness evaluations, the optimization process can become computationally intensive, even if one single evaluation is not particularly computationally expensive. The main contributions of this work are summarized as follows.

- An in-depth investigation of random forest (RF) assisted evolutionary optimization of expensive multi-objective constrained combinatorial problems is performed. This includes the scalability of the performance to the dimension of the search space, the influence of dimension reduction techniques and a comparison with the RBF-based surrogates. Note that research on RF-assisted evolutionary optimization has been reported for parameter tuning of a general algorithm [35] and surrogate-assisted genetic programming for symbolic regression [56], although the problems addressed therein are low-dimensional nonconstrained single-objective problems.

- Logistic regression models are introduced to rectify the non-dominated ranking according to the surrogates for the constraint functions, as it is found that the EA is more likely to be misled by the approximated constraint functions than the approximated objective functions in the problems studied in this work. Additionally, stochastic ranking based on two selection criteria [57] is employed to further reduce the influence of the approximated constraint functions.

- The performance of the proposed RF-assisted MOEA variants are studied in comparison with an RBF-assisted MOEA and three existing MOEAs without using surro- gates on the multi-objective knapsack problems (MOKPs) up to 100 decision variables. In addition, the proposed algorithms are applied to two real-world trauma system design problems, one involving 18 hospitals with 40,000 data records collected in Scotland and the other involving 72 hospitals with 100,000 records collected in Colorado in the US. The results show that the RF-assisted MOEA is able to achieve satisfactory non-dominated solutions using a limited computation budget.

The remainder of the paper is organized as follows. In Section II, the main components of the proposed algorithm are described in detail. Empirical results on MOKPs are presented and analyzed in Section III. Furthermore, we apply the proposed algorithm to two real-world trauma system design problems in Section IV. Section V concludes the paper.

\section{Proposed Algorithm}

\section{A. Constrained Multi-Objective Combinatorial Optimization Problems}

Generally, a combinatorial optimization problem with $m$ objectives and $h$ inequality constraints can be described as follows:

$$
\begin{gathered}
\min F(\mathbf{x})=\left(f_{1}(\mathbf{x}), \ldots, f_{m}(\mathbf{x})\right)^{T} \\
\text { s.t. } G(\mathbf{x})=\left(g_{1}(\mathbf{x}), \ldots, g_{h}(\mathbf{x})\right)^{T} \leq 0
\end{gathered}
$$

where $\mathbf{x}$ is an $n$-dimensional decision variable vector whose domain is a set of finite elements. The $m$ objectives are often conflicting with each other, thus there is no single ideal solution that can achieve the optimal value of all objectives. Like in continuous multi-objective optimization problems (MOPs), the optimal solution set is denoted as Pareto set (PS), whose corresponding objective values are called Pareto front (PF) [58]. Note that this work focuses on constrained multiobjective combinatorial optimization problems with two and three objectives.

\section{B. General Framework}

Over the past two decades, a large number of multi-objective optimization evolutionary algorithms (MOEAs) have been developed for solving MOPs with two or three objectives [59]. Generally, MOEAs can be divided into dominance based approaches, such as NSGA-II [60], decomposition based approaches, e.g., MOEA/D [61], and performance driven approaches [62]. To solve data-driven constrained multi-objective combinatorial optimization problems, this paper proposes an SAEA using the dominance based approach [60]. Since the objectives and constraints can be evaluated using historical data only, surrogates $(\hat{F}(\mathbf{x})$ for objective functions and $\hat{G}(\mathbf{x})$ for constraints) are constructed. For convenience, we call the algorithm using RF models [63] random forest assisted constrained multi-objective combinatorial optimization (RFCMOCO), and the algorithm using RF models after feature selection RF-CMOCO(FS). Since the RBF model is one of few surrogate models that have been adopted for data-driven combinatorial optimization problems [36], [37], we replace the $\mathrm{RF}$ model in RF-CMOCO with the RBF model, which is the third variant studied in this work called RBF-CMOCO. 


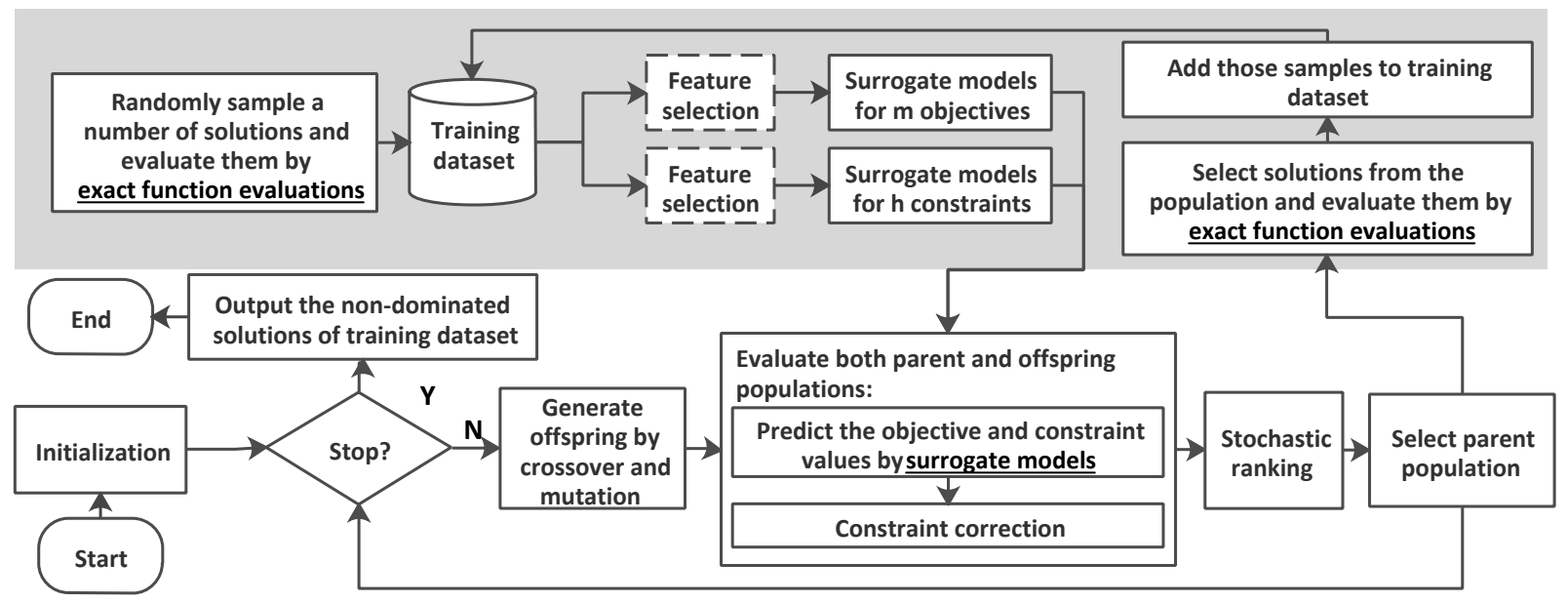

Fig. 1. A diagram of the proposed framework.

A diagram of the proposed framework is shown in Fig. 1. It consists of two main parts, an evolutionary optimizer and a model management strategy. Surrogate models for $m$ objectives and $h$ constraints are built separately on the basis of the training dataset, where a feature selection (FS) technique can be employed to reduce the dimension of decision space. The evolutionary optimizer searches for feasible optimal solutions based on the cheap surrogate models. An individual-based model management strategy [26] is adopted, where a number of promising solutions from the current population are selected and evaluated using the expensive objective and constraint functions. In SAEAs, solutions whose predicted fitness is better than the best solution found so far, or whose predicted fitness has a large degree of uncertainty are considered to be promising. These new data are then added to the training dataset for updating the surrogates. The proposed framework begins with a number of randomly sampled solutions (which are the initial training dataset for the surrogates) and finally outputs a set of non-dominated solutions. To reduce the impact resulting from the errors introduced by the approximated constraints, a logistic regression model is trained for each constraint to rectify the boundaries distinguishing feasible solutions from infeasible ones. Finally, the population is sorted based on stochastic ranking using two selection criteria, one based on the ranking as a constrained MOP, and the other on ranking as an unconstrained MOP.

\section{Surrogate Modeling}

1) Random Forest: Most surrogate models were designed for approximating continuous functions. Since the decision variables of combinatorial optimization problems are discrete, we use RF and RBF models as the surrogates to approximate the $m$ objectives and $h$ functions. Compared with RBF models, RF models have not been widely used as surrogates, although it has recently been suggested that the tree structure in RF models is well suited for approximating functions with discrete decision variables [34].

An RF model [63] is an ensemble of a large number of classification and regression trees (CARTs) [64], as illustrated in Fig. 2. Each CART is trained by different bootstrap samples, which is the reason why these $k$ trees have different structures in Fig. 2. Given $N$ training samples for an objective or a constraint function, $d=(2\lfloor\sqrt{n}\rfloor)$ decision variables are randomly selected from the $n$ total decision variables for the $N$ bootstrap samples of each tree [65]. Thus, one variable may be repeatedly included in a tree. The final output of the input $\mathrm{x}$ is the average of the outputs of $k$ trees. $k$ is set to 100 in RF-CMOCO and RF-CMOCO(FS) as recommended in [66].

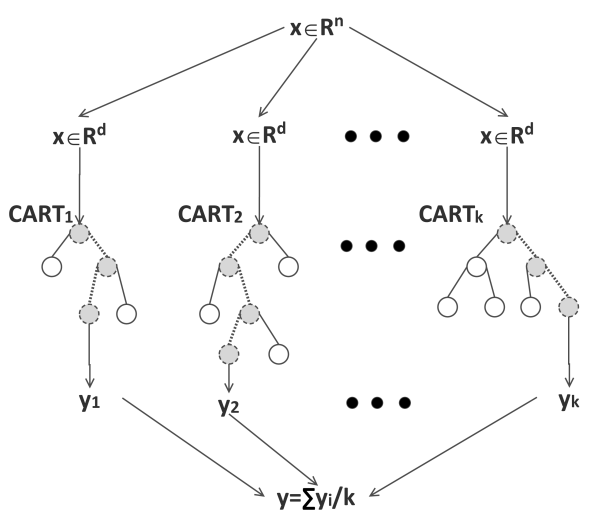

Fig. 2. An illustration of the random forest.

CART [64] has a binary tree structure, which is suited for the modeling discrete problems. CART divides the decision space into rectangle regions with leaf nodes, and the output of each leaf node is the average output of the samples in each divided region. Growing a CART is usually based on splitting and stopping criteria [67]. In RF-CMOCO and RFCMOCO(FS), the CART splits based on the minimization of the mean squared error (MSE) of the tree. It stops splitting when the reduction of the MSE is smaller than a pre-specified threshold, which is set to $1 e-4 * \sigma^{2}\left(\sigma^{2}\right.$ is the variance for the entire data before the CART is grown) in the empirical studies.

$m+h$ RF models for $m$ objective and $h$ constraint functions are built separately. For each RF models, $k$ CARTs are trained 
using $k$ sets of bootstrap samples. Thus, $k \times(m+h)$ CARTs are built for surrogate modeling in RF-CMOCO and RF$\mathrm{CMOCO}(\mathrm{FS})$.

2) Feature Selection: The amount of required data for training an RF model dramatically increases as the number of the decision variables increases. Therefore, we employ a feature selection technique for problems with more than 50 decision variables to reduce the input dimension before training the RF models for all $m$ objective and $h$ constraint functions. To this end, we employ the Kendall rank correlation coefficient (KRCC) [37] to measure the correlation between the decision variables and the objective or the constraint. Taking the $i$-th decision variable $x_{i}$ as an example, $N$ samples of $x_{i}$ and an objective in the training dataset are written as $\left\{\left(x_{i}^{1}, y^{1}\right), \ldots,\left(x_{i}^{N}, y^{N}\right)\right\}$. Any two samples $\left(x_{i}^{j}, y^{j}\right)$ and $\left(x_{i}^{k}, y^{k}\right)$ are compared. If $x_{i}^{j}>x_{i}^{k}$ and $y^{j}>y^{k}$, or if $x_{i}^{j}<x_{i}^{k}$ and $y^{j}<y^{k}$, the pair of samples is said to be concordant [68]. If $x_{i}^{j}>x_{i}^{k}$ and $y^{j}<y^{k}$, or if $x_{i}^{j}<x_{i}^{k}$ and $y^{j}>y^{k}$, the pair of samples is said to be discordant [68]. The KRCC $\left(\tau_{i}\right)$ between $x_{i}$ and the objective can be calculated as follows:

$$
\tau=\frac{n_{c}-n_{d}}{N(N-1) / 2}
$$

where $n_{c}$ is the number of concordant pairs and $n_{d}$ is the number of discordant pairs. $\tau_{i}$ ranges within $[-1,1]$, the closer $\tau_{i}$ approximates to -1 or 1 , the stronger $x_{i}$ is correlated to the objective. If $\tau_{i}=0, x_{i}$ and the objective are independent, namely, $x_{i}$ can be ignored when training the RF model for this particular objective or constraint.

Before training an RF model for approximating an objective or a constraint function, the KRCC between each decision variable and the objective or the constraint is calculated based on the training dataset. The contribution ratio of $x_{i}$ to the objective or the constraint is defined as:

$$
R_{i}=\frac{\left|\tau_{i}\right|}{\sum_{j=1}^{n}\left|\tau_{j}\right|}
$$

which is the ratio of $\left|\tau_{i}\right|$ to the sum of all the absolute KRCC values. The contribution ratios of all the decision variables are sorted in a descending order. The selection of decision variables is based on the sorted order, i.e., the decision variable with a larger contribution ratio will have a higher priority to be selected. In the proposed algorithm, $q$ decision variables are successively selected according to the sorting order until the accumulative contribution ratio of the selected decision variable amounts to $95 \%$. Thus, $q$ decision variables that are highly correlated with the objective or the constraint are retained. It is worth noting that $q$ is not a parameter to be predefined by the user, rather it is problem-dependant. After selecting $q$ decision variables, the setting for training the RF model changes accordingly. Thus, growing each CART is based on $2\lfloor\sqrt{q}\rfloor$ randomly selected decision variables.

3) Model Management: In SAEAs, the model management strategy is responsible for selecting individuals to be evaluated using the expensive objective functions and updating the surrogates. Most existing model management strategies have been developed for surrogate-assisted non-constrained optimization problems, which typically select solutions that can help accelerate convergence and/or promote diversity to strike a balance between exploitation and exploration.

In this work, an individual-based model management strategy is proposed to deal with constrained optimization, aiming to take into account both convergence and constraint handling. Like other model management strategies, a number of solutions will be randomly generated and evaluated using the expensive objective and constraint functions before optimization starts. All the randomly sampled solutions, no matter whether they are feasible or infeasible, are included in the initial training dataset for training the surrogates. Note, however, that the Latin hypercube sampling widely used for continuous optimization is not applicable for combinatorial optimization problems.

During the optimization, all offspring individuals at each generation will be evaluated at first using the surrogates. Then the individuals are sorted using the non-dominated sort [60], [69] according to degree of violation on the constraint functions $(\max \{G(\mathbf{x}), 0\})$. Based on this order, solutions are successively determined whether they are potentially better than the non-dominated solutions in the training dataset (denoted by $\left.P_{n d}\right)$, which are the best-so-far solutions. Recall that all objective values of the solutions in the current generation are estimated using the surrogates. To account for the approximation errors when comparing the solutions with those in $P_{n d}$, we calculate the root mean square errors (RMSEs) of $m$ RF models for the objectives, which are denoted as $\mathbf{E}_{n d}=\left\{e_{1}, \ldots, e_{m}\right\}$. Then, we use these errors to estimate the upper bound of the objective values of the solutions in the current generation. Let the estimated objective values of solution $\mathbf{x}$ be $\hat{F}(\mathbf{x})$, then the upper bound (best possible) objective values in case of minimization are assumed to be $\hat{F}(\mathbf{x})-\mathbf{E}_{n d}$. If no solution in $P_{n d}$ is able to dominate solution $\mathbf{x}$ according to its estimated best objective values, solution $\mathbf{x}$ will then be selected for evaluation using the expensive real fitness functions. The model management strategy is also outlined in Algorithm 1.

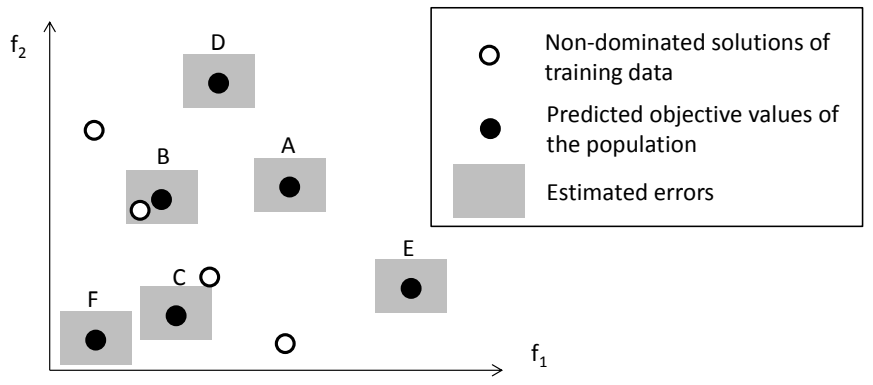

Fig. 3. An illustration of the model management strategy for a 2-objective problem, where $f_{1}$ and $f_{2}$ are two objectives.

Fig. 3 provides an illustrative example of a 2-objective problem, where the circles denote the non-dominated solutions in the training data $\left(P_{n d}\right)$ and the dots $(\mathrm{A}-\mathrm{F})$ denote the individuals in the current population evaluated using the surrogates. Due to the approximation errors of the RF models, the true objective values of these solutions are indicated by the shaded rectangle and the best possible values are 


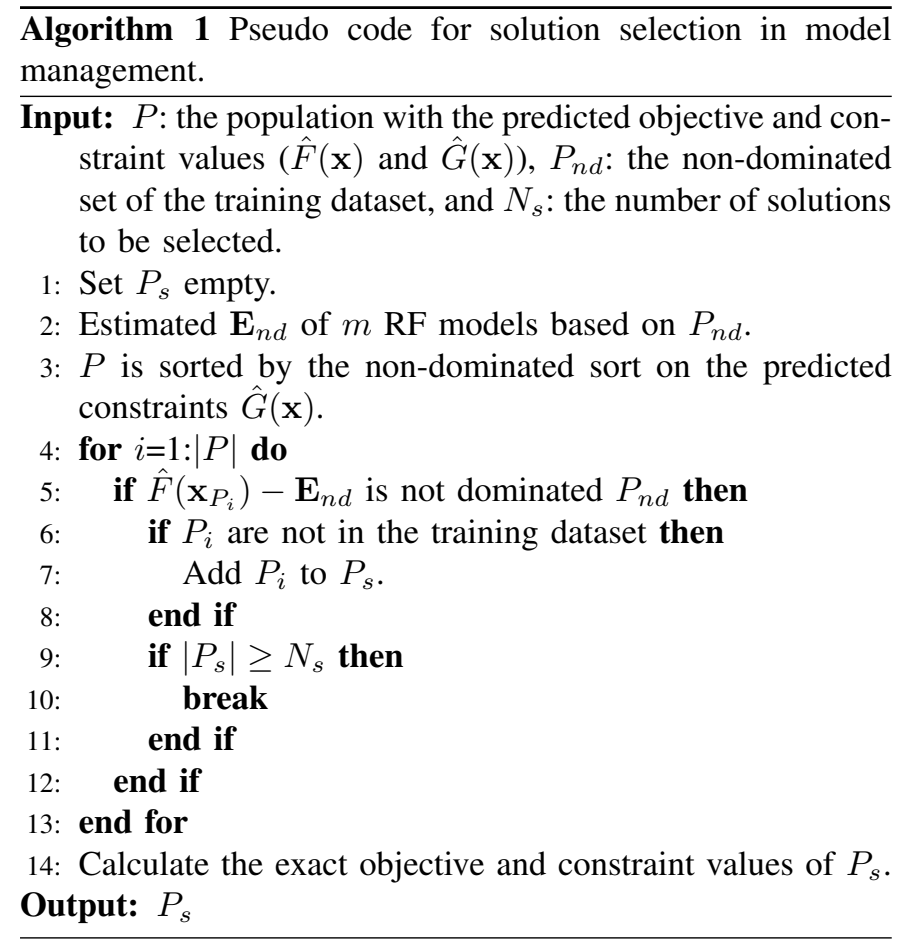

located at the bottom-left corner of the rectangle. Assume we intend to select two solutions from solutions $\mathrm{A}$ to $\mathrm{F}$ to be evaluated using the expensive objective functions. At first, the management strategy performs a non-dominated sort of the six solutions based on their predicted constraints $\hat{G}(\mathbf{x})$. Assume the resulting order is A, B, C, D, E and F, starting from the best. Now the sorted solutions are checked one by one to see whether they will be selected. Although solution A ranks the first, it is not able to dominate any solutions in $P_{n d}$ and consequently, solution A will not be selected. Then solution B is checked, which is found to be able to dominate one solution in $P_{n d}$ by taking the estimation error into account (otherwise B will not be selected either). Solution $\mathrm{C}$ will also be selected as it is able to dominate one solution in $P_{n d}$. Thus at this generation, solutions $\mathrm{B}$ and $\mathrm{C}$ are selected. Note that solution $\mathrm{F}$ is not selected since two solutions have already been chosen, even if it is the best solution according to the estimated objective values.

The proposed model management strategy hypothesizes that if the best solutions are far away from the infeasible region, convergence should be prioritized and therefore the better solutions should be selected. However, if the number of feasible solutions is very small, indicating the population is close to the infeasible region, constraint handling should be more important. In this case, infeasible solutions closest to the boundaries should be selected. Finally, the approximation errors are estimated by evaluating the surrogates using the non-dominated solutions in the training data so that potentially better solutions are selected and evaluated using the expensive objective and constraint functions.

\section{Constraint Handling}

In this work, constraints are approximated using surrogate models. Due to the approximation errors, some infeasible solutions may be treated to be feasible, which will seriously mislead the evolutionary search. To mitigate this problem, we propose two novel constraint handling strategies in this section.

1) Logistic Regression for Constraint Correction: To reduce the possibility of classifying feasible solutions to be infeasible, the first strategy we propose aims to rectify the boundaries between the feasible and infeasible regions defined by the surrogate constrained functions.

Take the situation illustrated in Fig. 4 as an example. In the figure, the solutions denoted by the plus signs are feasible ones and those by the cross signs are infeasible. The $j$-th constraint $g_{j}(\mathbf{x})$ is approximated by the surrogate model $\hat{g}_{j}(\mathbf{x})$. Because of approximation errors, two feasible solutions are classified as infeasible. If the constraint $\hat{g}_{j}(\mathbf{x}) \leq 0$ is changed to $\hat{g}_{j}(\mathbf{x}) \leq$ $\alpha_{j}$, where $\alpha_{j}$ is the boundary between feasible and infeasible solutions, the misclassified solutions can be corrected.

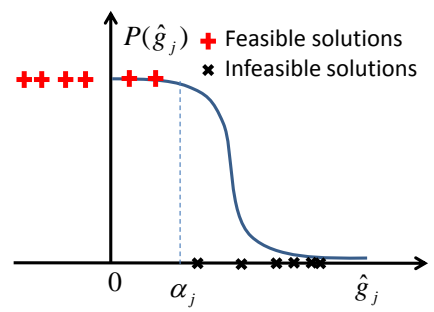

Fig. 4. An illustration of using logistic regression for constraint correction.

To rectify the boundary, we use a logistic regression model [70] to estimate the probability at which solution $\mathbf{x}$ with the approximated constraint value $\hat{g}_{j}(\mathbf{x})$ is feasible. The logistic regression model can be described as follows:

$$
P\left(\hat{g}_{j}\right)=\frac{1}{1+e^{\beta_{0}+\beta_{1} \hat{g}_{j}}}
$$

where $\beta_{0}$ and $\beta_{1}$ are two parameters to be estimated. The training dataset is used to estimate the logistic regression model, where the predicted value $\hat{g}_{j}(\mathbf{x})$ of each sample is the input, and the probability of the solution being feasible is the output $\left(\hat{g}_{j}(\mathbf{x}) \leq 0\right.$ is set to 1 and $\hat{g}_{j}(\mathbf{x})>0$ is set to 0$)$. Solutions are believed to be feasible for constraint $\hat{g}_{j}$ when $P\left(\hat{g}_{j}\right) \geq 0.95$. Thus, the new boundary $\alpha_{j}$ is defined as $P\left(\alpha_{j}\right)=0.95$, the constraint $g_{j}(\mathbf{x}) \leq 0$ is changed to $\hat{g}_{j}(\mathbf{x}) \leq \alpha_{j}$.

In each generation, after $h$ surrogate models $\hat{g}_{j}(\mathbf{x})(1 \leq$ $j \leq h)$ are built, their feasible probabilities are learned by $h$ logistic regression models described in Eq. (4), then the boundaries $\alpha_{j}$ are calculated. For the following selection, the problem is changed to Eq. (5) with $\alpha_{j}$ offset on the constraints.

$$
\begin{gathered}
\min \hat{F}(\mathbf{x})=\left(\hat{f}_{1}(\mathbf{x}), \ldots, \hat{f}_{m}(\mathbf{x})\right)^{T} \\
\text { s.t. } \hat{G}(\mathbf{x})=\left(\hat{g}_{1}(\mathbf{x})-\alpha_{1}, \ldots, \hat{g}_{h}(\mathbf{x})-\alpha_{h}\right)^{T} \leq 0
\end{gathered}
$$

2) Stochastic Ranking Using Two Selection Criteria: It has been shown that the performance of handling constraints in SAEAs can be improved by converting constrained MOPs into unconstrained MOPs [71], [72], namely, constraints in Eq. (1) can be seen as additional objectives [44] as follows:

$$
F^{*}(\mathbf{x})=\min \left(f_{1}(\mathbf{x}), \ldots, f_{m}(\mathbf{x}), g_{1}(\mathbf{x}), \ldots, g_{h}(\mathbf{x})\right)^{T}
$$


Thus, two selection criteria are available for sorting the population: ranking as an unconstrained MOP having $m+h$ objectives, or as a constrained MOP having $m$ objectives and $h$ inequality constraints. A variety of efficient non-dominated sorting techniques can be used [69], [73].

Stochastic ranking [57] is a bubble-sort-like procedure, which was proposed to strike a balance between searching for better objectives and finding feasible solutions. In the original version of stochastic ranking, swapping adjacent solutions is based either on the objective values or on the constraint values at a certain probability. However, we adopt stochastic ranking in the proposed algorithm to balance two selection criteria, thereby further reducing the influence the approximation errors introduced by the approximated constraints.

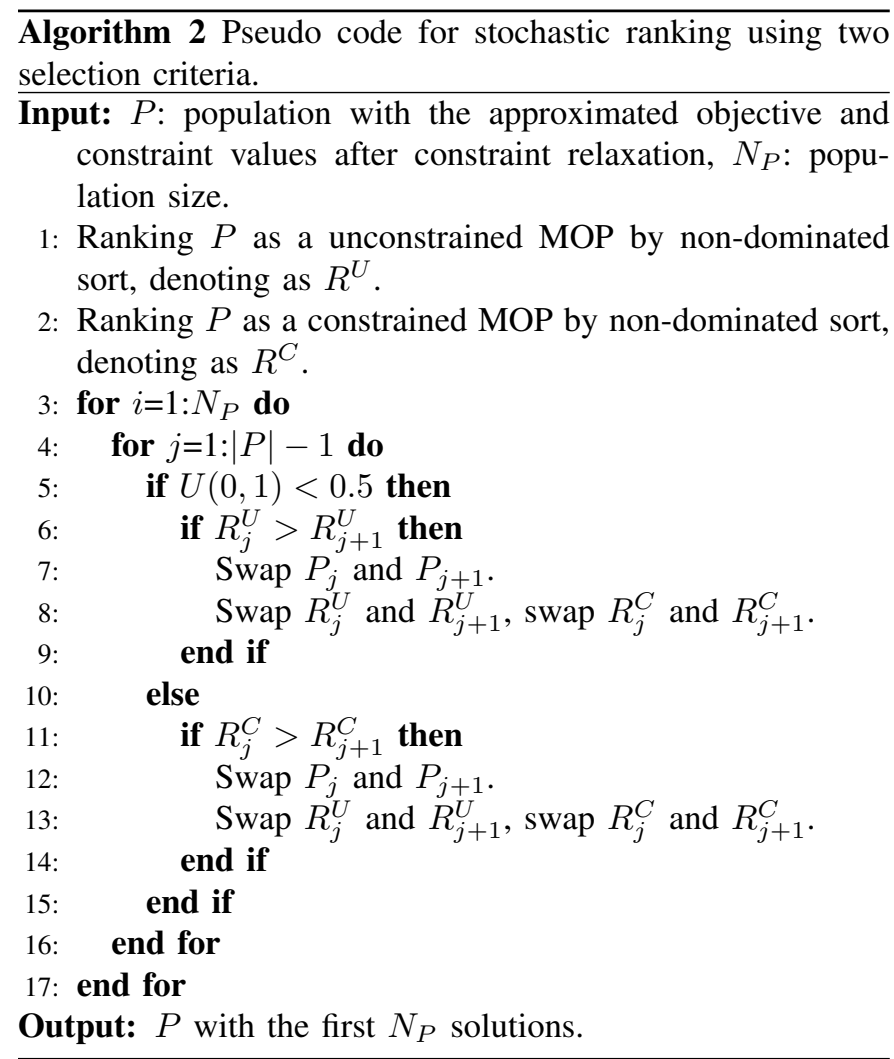

The stochastic ranking process in the proposed algorithm are described in Algorithm 2, where $U(0,1)$ is a random number in $[0,1]$. At each generation, a combination of the parent and offspring populations $P$ is evaluated using the surrogate models, where the constraint boundaries have already been rectified using the logistic regression models. Before performing the stochastic ranking, $P$ is ranked as an unconstrained MOP with $m+h$ objectives and a constrained MOP with $m$ objectives and $h$ inequality constraints, respectively, the assigned ranks are denoted as $R^{U}$ and $R^{C}$. In the bubble-sort, the comparisons between two adjacent solutions are based either on $R^{U}$ or on $R^{C}$ at a probability of 0.5 . Once the stochastic bubble-sort terminates, the first $N_{P}$ solutions in $P$ are selected as the parents of the next generation.

\section{E. Detailed Settings}

The proposed framework involves surrogate modeling, optimization, and constraint handling. For clarify, we summarize the parameter settings for the three variants of the proposed algorithm, RF-CMOCO, RF-CMOCO(FS), and RBF-CMOCO in Table I.

TABLE I

PARAMETER SETTINGS FOR RF-CMOCO, RF-CMOCO(FS), AND $\mathrm{RBF}-\mathrm{CMOCO}$

\begin{tabular}{|c|c|c|}
\hline Component & $\begin{array}{l}\text { Parameter/ } \\
\text { Techique }\end{array}$ & Settings \\
\hline \multirow{6}{*}{ Surrogate } & $\begin{array}{l}\text { Initial } \\
\text { dataset }\end{array}$ & 1000 random solutions \\
\hline & $\mathrm{RF}$ & $\begin{array}{l}100 \text { CARTs that generated from the boot- } \\
\text { strap samples of } 2\lfloor\sqrt{n}\rfloor \text { randomly selected } \\
\text { decision variables }\end{array}$ \\
\hline & CART & $\begin{array}{l}\text { Splitting tolerance is set to } 1 e-4 * \sigma^{2} \text { ( } \sigma^{2} \\
\text { is the variance for the entire data) }\end{array}$ \\
\hline & $\mathrm{RBF}$ & $\begin{array}{l}n \text { hidden nodes using the overlap mea- } \\
\text { sure [74] as the kernel function }\end{array}$ \\
\hline & FS & $\begin{array}{l}\text { Top } q \text { decision variables with accumulative } \\
\text { KRCC contribution ratio of } 95 \%\end{array}$ \\
\hline & $N_{s}$ & $\begin{array}{l}5 \text { exact function evaluations in each gener- } \\
\text { ation }\end{array}$ \\
\hline \multirow{2}{*}{ Optimization } & Population & 100 solutions \\
\hline & Budget & 1500 or 2000 exact function evaluations \\
\hline $\begin{array}{l}\text { Constraint } \\
\text { handling }\end{array}$ & $\begin{array}{l}\text { Probability } \\
\text { of feasibility }\end{array}$ & 0.95 \\
\hline
\end{tabular}

\section{EXPERIMENTAL STUDIES}

Unlike MOPs with continuous decision variables, multiobjective combinatorial optimization problems may have different structures (or presentation) of decision variables [75]. For example, MOKPs have categorical decision variables, while multi-objective flow shop scheduling problems have permutation decision variables. For some structures like permutation decision variables, surrogate models cannot be directly used, and mapping- or similarity-based methods need to be applied [34]. Since the use of indirect surrogate models is out of the scope of this work, we choose MOKPs as the test problem, which can be generated by using the method reported in [76]. An MOKP with $m$ objectives and $n$ items can be modeled as follows:

$$
\begin{aligned}
& \max f_{i}=\sum_{j=1}^{n} v_{j}^{i} x_{j}, x_{j} \in\{0,1\}, 1 \leq i \leq m \\
& \text { s.t. } \sum_{j=1}^{n} w_{j} x_{j} \leq W
\end{aligned}
$$

where $m$ objectives are the total values of the chosen items, $w_{j}$ and $v_{j}^{i}$ are the weights and value for $f_{i}$ of the $j$-th item, and $W$ is the weight limitation of the knapsack. Similar to the generation method in [76], $w_{j}$ and $v_{j}^{i}$ are random integers within $[1,1000]$, and $W$ is set to $0.5 \sum_{j=1}^{n} w_{j}$. Based on the above method, we generate MOKP instances with 10 to 100 items and 2 to 3 objectives ${ }^{1}$. Here we assume each evaluation in Eq. (7) is expensive. In this section, a fixed number of those expensive evaluations are set as the allowed computational budget of the compared algorithms.

\footnotetext{
${ }^{1}$ https://sites.google.com/site/handingwanghomepage/downloading
} 


\section{A. Approximation Performance of Random Forest Models}

We first investigate the approximation performance of RF models for MOKPs. As the number of decision variables may heavily affect the performance of surrogates, we choose the first objectives of the 20-item MOKP as a low-dimensional test case and the 100-item MOKP as a high-dimensional test case. The experiment is conducted on the RF models with and without the feature selection for 30 independent times, where 100 to 2500 random solutions serve as the training dataset and 10,000 random solutions as the test dataset. In the experiment, we use the same settings for the RF models as recommended in [65], [66], which are presented in Table I.

The average RMSEs of the two RF models on the test dataset over the size of the training data are plotted in Fig. 5. From the figure, we can see that the performance of both RF models on 20- and 100-item MOKP instances enhances as the number of the training data increases. In the beginning, RMSEs of the two RF models drop rapidly when new data are added to the training dataset. However, the decrease of RMSEs becomes slow as the size of the training dataset further increases. In addition, it is noticed that feature selection can significantly enhance the performance of the RF model on the 100-item MOKP instance, but worsens the performance on the 20-item MOKP instance. These results indicate that feature selection significantly improves the performance of RF models in approximating functions with a large number of discrete variables.
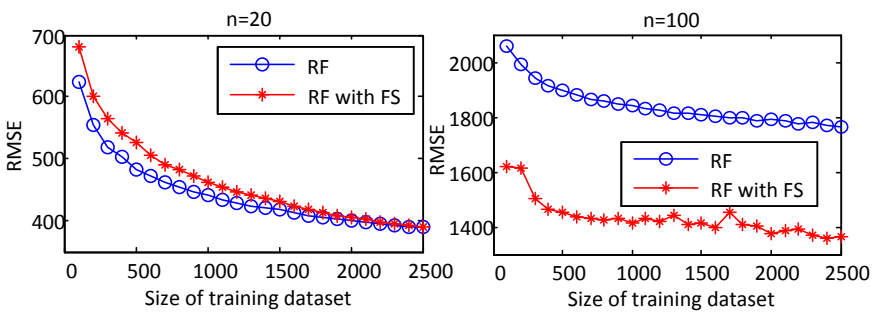

Fig. 5. Average RMSEs of the RF models over the size of the training data with and without feature selection on two-objective MOKP instances with 20 and 100 items.

\section{B. Constraint Handling Strategies}

As shown in Fig. 5, the RMSE of the RF model remains very large even though the size of the training dataset has been increased to 2500 , making it hard to distinguish feasible solutions from infeasible ones using the surrogate models. Therefore, additional constraint handling strategies are designed in this work. In this subsection, we evaluate the effectiveness of the proposed constraint handling strategies by performing experiments on a bi-objective MOKP instance with 20 items so that the influence of the objectives is minimized. As the experiments are conducted on the 20-item MOKP instance, no feature selection is applied in building the RF models.

The first strategy is to use logistic regression models to rectify the constraint boundaries. To examine the effectiveness of this strategy, we compare the performance of the RF models with and without the constraint correction strategy using the
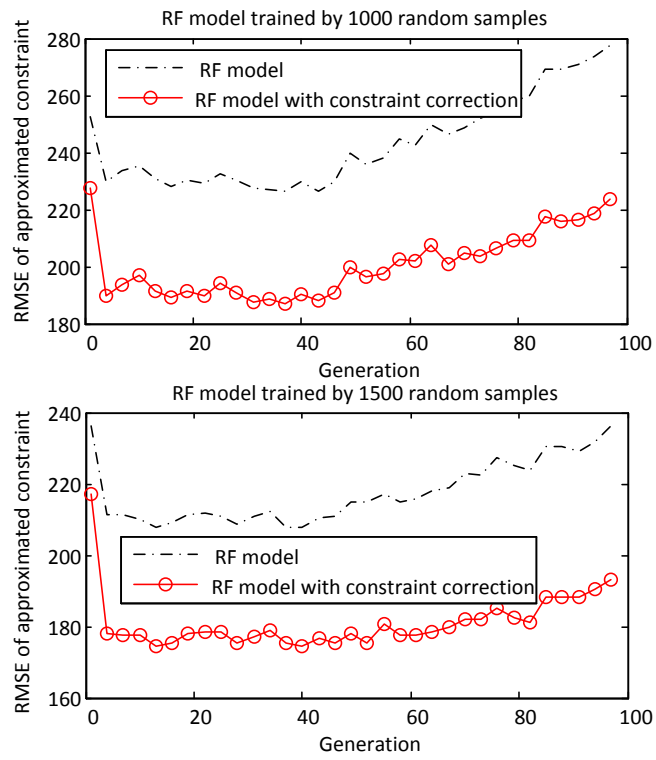

Fig. 6. Average RMSEs of the RF models with and without constraint correction on the data generated by NSGA-II optimizing the bi-objective MOKP instance with 20 items.

data generated by NSGA-II of a population size 100. The experimental details are described in the following.

- Test datasets generation: Run the NSGA-II using exact function evaluations on the MOKP instance for 30 independent times, where the population size is set to 100 and a maximum of 100 generations are run. The 100 solutions generated in each generation are recorded as a dataset for evaluating the constraint handling strategies. Thus, 100 datasets are stored in each run.

- Constraint correction: For the 100 datasets generated in each run, two RF models are built from 1000 and 1500 random samples separately, then the boundaries separating the feasible and infeasible solutions $\alpha$ based on the two different strategies are calculated. Recall that the proposed constraint correction strategy changes their prediction of constraints to $\hat{G}(\mathbf{x})-\alpha$.

- Performance evaluation: Predict the constraint values in every generation by the RF models with and without constraint correction. The RMSEs of the RF models are calculated for the solutions generated in each of the 100 generations.

The average RMSEs of the RF models with and without constraint correction in different generations are shown in Fig. 6. As the search proceeds, the population (the test dataset) becomes concentrated, the RMSE in local areas gradually increases. It can be observed that RF models trained using 1500 samples have a smaller RMSE than those trained using 1000 samples. It can also be seen that the constraint correction strategy reduces the RMSEs of both RF models. These results indicate that the logistic regression based correction strategy is able to improve the performance of the RF models of the constraint functions.

The second strategy we employ is a stochastic ranking strategy using two selection criteria to further reduce the influence of the approximation errors induced by the RF 
models. We conduct the following experiments to demonstrate the effects of the stochastic ranking strategy.

- Generation of the test datasets: Run the NSGA-II using exact function evaluations on the MOKP instance for 30 independent times, where the population size and the terminal criterion are set as in the previous experiments. The combination of parent and offspring populations and the selected population $P_{s}$ in each generation are recorded as the datasets for evaluating the stochastic ranking strategy.

- Prediction via surrogate models: For the 100 datasets generated in each run, two RF models are built using 1000 and 1500 random samples separately. Then the constraint correction strategy is applied on both models. Predict the objective and constraint values of the combined population in each generation using the RF models with the constraint correction strategy.

- Selection: For each generation, select populations $\left(P_{s}^{U}\right.$, $P_{s}^{C}$, and $P_{s}^{S R}$ ) from the combined population using three different ranking strategies, namely, ranking by considering the MOKP as an unconstrained MOP, a constrained MOP, or the proposed stochastic ranking.

- Performance evaluation: Calculate the percentages of $P_{s}^{U} \cap P_{s}, P_{s}^{C} \cap P_{s}$, and $P_{s}^{S R} \cap P_{s}$ in $P_{s}$ as the selection accuracy.
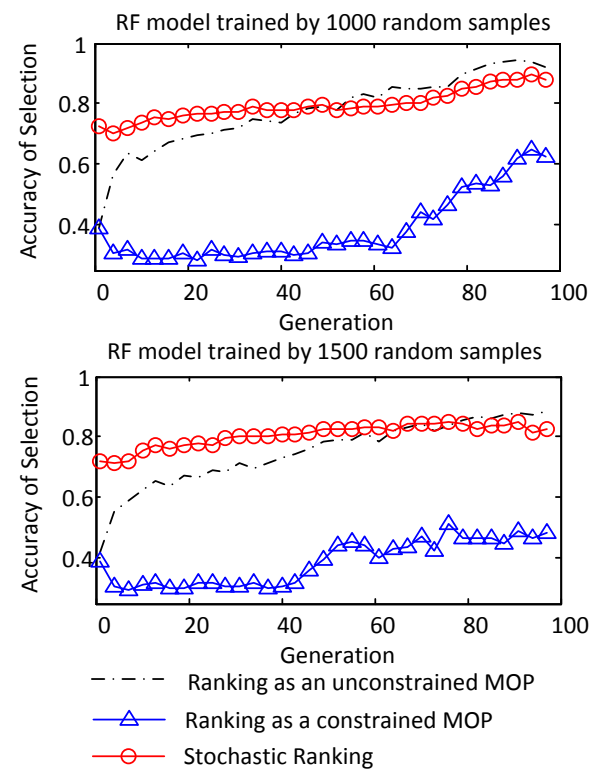

Fig. 7. Average selection accuracy of the three different ranking strategies based on the RF models trained using 1000 and 1500 random samples on a bi-objective MOKP instance with 20 items.

For the MOKP instance, the average selection performance of the three different ranking methods based on the RF models trained using 1000 and 1500 random samples in different generations are shown in Fig. 7. It can be seen that for both RF models, the selection accuracy of the ranking method considering the problem as a constrained MOP is about half of that of the ranking method considering the problem as an unconstrained MOP. However, stochastic ranking, which is a hybrid of these two ranking methods, is able to improve the selection accuracy to 0.7 . In particular in the early generations, the stochastic ranking can achieve much higher selection accuracy than the other two ranking strategies. As the search proceeds, however, the percentage of feasible solutions in the population gradually increases, the performance of the ranking strategy considering the problem as an unconstrained MOP rapidly improves and even becomes slightly better at the later generations. Nevertheless, the stochastic ranking maintains an overall satisfactory selection accuracy in the entire search process of NSGA-II. We also find that the selection based on the RF model trained using 1500 data samples performs slightly better, due to better-trained models.

\section{Comparative Experiments}

In this section, RF-CMOCO, RF-CMOCO(FS), and RBF$\mathrm{CMOCO}$ are compared with other algorithms on two- or three-objective MOKP instances with 10 to 100 items. Both mathematical programming and MOEAs have been employed for solving MOKPs [3]. The algorithms using mathematical programming [77] require analytic functions of MOKPs, which does not meet the assumption that analytic objective and constraint functions are not available made in this work. Therefore, we choose two MOEAs for comparison.

- Generic MOEAs: NSGA-II [60] and MOEA/D [61] are two popular MOEAs without using any domain knowledge or local search strategy.

- Specific MOEAs: Variable neighborhood search (VNS) has been shown effective in solving MOKPs in [78], although analytic functions are used. For fair comparison, we include MO-GVNS [79], an MOEA with general VNS without using analytic functions, for comparison.

In the experiments, a maximum of 1500 exact fitness evaluations is allowed, of which 1000 fitness evaluations are used for building the RF models before the optimization starts and 500 exact fitness evaluations can be used during the optimization. All compared algorithms perform 30 independent times and they terminate when the allowed computation budget is exhausted. All algorithms under comparison use 3-point crossover with a probability of 1 and point mutations with a probability of 0.2 . In the experiments, the settings of RFCMOCOs and RBF-CMOCO are presented in Table I. The population size of MO-GVNS and NSGA-II is set to 100. The population size of MOEA/D (with the neighborhood parameter $T=30$ ) is set to 100 for 2-objective MOKPs and 105 for 3-objective MOKPs.

To evaluate the results obtained by the compared algorithms, inverted generational distance (IGD) [80], the average distance from a reference set to the obtained solution set, is adopted, which is believed to be able to account for both convergence and diversity. We use the non-dominated set of solutions obtained from 30 runs of NSGA-II (with a population size of 100 run for 200 generations using exact function evaluations) as the reference set for calculating IGD. Further, we normalize the objectives using the extreme points in the reference set. The IGD values of the compared algorithms on the MOKP instances are presented in Table II, where the comparisons between RF-CMOCOs (with and without feature selection) 
TABLE II

THE IGD VALUES OF RF-CMOCOS (WITH AND WITHOUT FEATURE SELECTION), RBF-CMOCO, NSGA-II, MOEA/D, AND MO-GVNS ON THE MOKP INSTANCES WITH $n$ ITEMS $m$ OBJECTIVES. THE BEST AND SECOND BEST FITNESS VALUES AMONG ALL THE COMPARED ALGORITHMS FOR EACH INSTANCE ARE HIGHLIGHTED IN GRAY AND LIGHT GRAY. THE RESULTS OF RF-CMOCO VARIANTS AND OTHER COMPARED ALGORITHMS ARE ANALYZED USING THE WILCOXON SIGNED-RANK TEST (SIGNIFICANCE LEVEL=0.05). THE ANALYSIS RESULTS OF RF-CMOCO IS DENOTED AS THE SUPERSCRIPT IN BRACKETS, WHILE THOSE OF RF-CMOCO(FS) ARE DENOTED AS THE SUBSCRIPT IN BRACKETS.

\begin{tabular}{|c|c|c|c|c|c|c|c|}
\hline$n$ & $m$ & RF-CMOCO & RF-CMOCO(FS) & RBF-CMOCO & NSGA-II & MOEA/D & MO-GVNS \\
\hline 10 & 2 & $0.00 \mathrm{e}+00 \pm 0.00 \mathrm{e}+00(\stackrel{\bar{\approx}}{)})$ & $0.00 \mathrm{e}+00 \pm 0.00 \mathrm{e}+00(\stackrel{\approx}{=})$ & $1.11 \mathrm{e}-01 \pm 1.11 \mathrm{e}-01\left({ }_{+}^{+}\right)$ & $5.21 \mathrm{e}-02 \pm 1.09 \mathrm{e}-01(\underset{\approx}{\approx})$ & $2.52 \mathrm{e}-01 \pm 1.49 \mathrm{e}-01\left(_{+}^{+}\right)$ & $4.68 \mathrm{e}-02 \pm 9.59 \mathrm{e}-02(\underset{\approx}{\approx})$ \\
\hline 10 & 3 & $0.00 \mathrm{e}+00 \pm 0.00 \mathrm{e}+00(\stackrel{\stackrel{\equiv}{\approx}}{)})$ & $0.00 \mathrm{e}+00 \pm 0.00 \mathrm{e}+00(\stackrel{\approx}{=})$ & $3.63 \mathrm{e}-02 \pm 2.48 \mathrm{e}-02\left(_{+}^{+}\right)$ & $2.28 \mathrm{e}-02 \pm 1.85 \mathrm{e}-02\left({ }_{+}^{+}\right)$ & $1.26 \mathrm{e}-01 \pm 3.95 \mathrm{e}-02\left(_{+}^{+}\right)$ & $0.00 \mathrm{e}+00 \pm 0.00 \mathrm{e}+00(\stackrel{\approx}{\approx})$ \\
\hline 20 & 2 & $1.11 \mathrm{e}-01 \pm 4.74 \mathrm{e}-02(\stackrel{\approx}{\approx})$ & $1.36 \mathrm{e}-01 \pm 3.84 \mathrm{e}-02(\stackrel{\approx}{=})$ & $2.86 \mathrm{e}-01 \pm 6.59 \mathrm{e}-02\left({ }_{+}^{+}\right)$ & $4.26 \mathrm{e}-01 \pm 6.84 \mathrm{e}-02\left({ }_{+}^{+}\right)$ & $5.67 \mathrm{e}-01 \pm 8.81 \mathrm{e}-02\left({ }_{+}^{+}\right)$ & $3.18 \mathrm{e}-01 \pm 7.05 \mathrm{e}-02\left({ }_{+}^{+}\right)$ \\
\hline 20 & 3 & $2.13 \mathrm{e}-01 \pm 3.30 \mathrm{e}-02(\stackrel{\bar{\approx}}{\approx})$ & $2.12 \mathrm{e}-01 \pm 3.68 \mathrm{e}-02(\stackrel{\widetilde{\approx}}{=})$ & $3.03 \mathrm{e}-01 \pm 4.88 \mathrm{e}-02\left({ }_{+}^{+}\right)$ & $5.35 \mathrm{e}-01 \pm 9.81 \mathrm{e}-02\left({ }_{+}^{+}\right)$ & $6.48 \mathrm{e}-01 \pm 1.24 \mathrm{e}-01\left({ }_{+}^{+}\right)$ & $3.92 \mathrm{e}-01 \pm 5.68 \mathrm{e}-02\left({ }_{+}^{+}\right)$ \\
\hline 30 & 2 & $2.81 \mathrm{e}-01 \pm 7.88 \mathrm{e}-02(\stackrel{\stackrel{=}{\approx}}{)})$ & $2.86 \mathrm{e}-01 \pm 5.66 \mathrm{e}-02(\stackrel{\approx}{=})$ & $5.07 \mathrm{e}-01 \pm 1.51 \mathrm{e}-01\left({ }_{+}^{+}\right)$ & $9.91 \mathrm{e}-01 \pm 1.56 \mathrm{e}-01\left({ }_{+}^{+}\right)$ & $1.08 \mathrm{e}+00 \pm 2.26 \mathrm{e}-01\left(\begin{array}{c}+ \\
+\end{array}\right)$ & $7.28 \mathrm{e}-01 \pm 1.43 \mathrm{e}-01\left({ }_{+}^{+}\right)$ \\
\hline 30 & 3 & $2.61 \mathrm{e}-01 \pm 3.13 \mathrm{e}-02(\stackrel{\bar{\approx}}{)})$ & $2.34 \mathrm{e}-01 \pm 2.99 \mathrm{e}-02(\stackrel{\widetilde{\approx}}{=})$ & $3.30 \mathrm{e}-01 \pm 3.14 \mathrm{e}-02\left({ }_{+}^{+}\right)$ & $5.38 \mathrm{e}-01 \pm 7.87 \mathrm{e}-02\left({ }_{+}^{+}\right)$ & $6.16 \mathrm{e}-01 \pm 1.15 \mathrm{e}-01\left({ }_{+}^{+}\right)$ & $4.41 \mathrm{e}-01 \pm 6.42 \mathrm{e}-02\left({ }_{+}^{+}\right)$ \\
\hline 50 & 2 & $2.28 \mathrm{e}-01 \pm 5.20 \mathrm{e}-02(\stackrel{\bar{\approx}}{\approx})$ & $2.38 \mathrm{e}-01 \pm 5.51 \mathrm{e}-02(\stackrel{\approx}{=})$ & $4.01 \mathrm{e}-01 \pm 7.65 \mathrm{e}-02\left({ }_{+}^{+}\right)$ & $7.26 \mathrm{e}-01 \pm 1.62 \mathrm{e}-01\left({ }_{+}^{+}\right)$ & $8.46 \mathrm{e}-01 \pm 1.21 \mathrm{e}-01\left({ }_{+}^{+}\right)$ & $5.68 \mathrm{e}-01 \pm 1.05 \mathrm{e}-01\left({ }_{+}^{+}\right)$ \\
\hline 50 & 3 & $2.72 \mathrm{e}-01 \pm 2.47 \mathrm{e}-02(\stackrel{\bar{\approx}}{)})$ & $2.58 \mathrm{e}-01 \pm 1.95 \mathrm{e}-02(\stackrel{\approx}{=})$ & $3.13 \mathrm{e}-01 \pm 2.84 \mathrm{e}-02\left({ }_{+}^{+}\right)$ & $4.88 \mathrm{e}-01 \pm 5.11 \mathrm{e}-02\left({ }_{+}^{+}\right)$ & $5.68 \mathrm{e}-01 \pm 6.27 \mathrm{e}-02\left({ }_{+}^{+}\right)$ & $3.60 \mathrm{e}-01 \pm 5.11 \mathrm{e}-02\left({ }_{+}^{+}\right)$ \\
\hline 100 & 2 & $4.02 \mathrm{e}-01 \pm 9.56 \mathrm{e}-02(\stackrel{=}{=})$ & $3.39 \mathrm{e}-01 \pm 6.73 \mathrm{e}-02(\stackrel{+}{=})$ & $4.67 \mathrm{e}-01 \pm 1.18 \mathrm{e}-01(\stackrel{\underset{+}{\approx}}{)})$ & $7.53 \mathrm{e}-01 \pm 1.61 \mathrm{e}-01\left({ }_{+}^{+}\right)$ & $9.35 \mathrm{e}-01 \pm 1.89 \mathrm{e}-01\left({ }_{+}^{+}\right)$ & $7.02 \mathrm{e}-01 \pm 9.57 \mathrm{e}-02\left({ }_{+}^{+}\right)$ \\
\hline 100 & 3 & $4.02 \mathrm{e}-01 \pm 5.23 \mathrm{e}-02(=)$ & $3.97 \mathrm{e}-01 \pm 5.70 \mathrm{e}-02(\stackrel{+}{=})$ & $4.57 \mathrm{e}-01 \pm 5.47 \mathrm{e}-02\left({ }_{+}^{+}\right)$ & $6.82 \mathrm{e}-01 \pm 1.18 \mathrm{e}-01\left({ }_{+}^{+}\right)$ & $7.39 \mathrm{e}-01 \pm 1.03 \mathrm{e}-01\left({ }_{+}^{+}\right)$ & $5.81 \mathrm{e}-01 \pm 7.95 \mathrm{e}-02\left({ }_{+}^{+}\right)$ \\
\hline
\end{tabular}

${ }^{+}$denotes the peer algorithm is significantly worse than RF-CMOCO or RF-CMOCO(FS). ${ }^{-}$denotes the peer algorithm is significantly better than RF-CMOCO or RF-CMOCO(FS),

$\approx$ denotes the peer algorithm is not significantly different from RF-CMOCO or RF-CMOCO(FS). $=$ denotes the peer algorithm is RF-CMOCO or RF-CMOCO(FS).

and other compared algorithms are also analyzed using the Wilcoxon signed-rank test [81].

From these results, we can see that MOEA/D performs the worst on all MOKP instances considered in the comparisons, which might be attributed to various reasons. Firstly, MOEA/D is in general not well suited for constrained optimization problems [82]. Without properly handling the constraints, MOEA/D is less efficient especially when a limited number of function evaluations are allowed. Furthermore, the true $\mathrm{PFs}$ of combinatorial MOPs are usually discontinuous and irregular, while MOEA/D generates weights evenly across the whole objective space. By contrast, NSGA-II is based on dominance comparison and is able to perform more robustly than MOEA/D when the PF of the MOPs are irregular or unknown. MO-GVNS performs better than NSGA-II, as it is designated for MOKPs and uses VNS for local search. However, MO-GVNS performs worse than RF-CMOCO, RF$\mathrm{CMOCO}(\mathrm{FS})$, or RBF-CMOCO, confirming that the use of surrogates is able to speed up the search.

TABLE III

THE RUNNING TIME(S) OF RF-CMOCOS (WITH AND WITHOUT FEATURE SELECTION) AND RBF-CMOCO ON THE MOKP INSTANCES WITH $n$ ITEMS $m$ OBJECTIVES. THE FASTEST AND SECOND FASTEST COMPARED ALGORITHMS ON EACH TEST PROBLEM ARE HIGHLIGHTED IN GRAY AND LIGHT GRAY.

\begin{tabular}{ccccc}
\hline$n$ & $m$ & RF-CMOCO & RF-CMOCO(FS) & RBF-CMOCO \\
\hline 10 & 2 & $2.90 \mathrm{e}+03 \pm 3.83 \mathrm{e}+02$ & $2.29 \mathrm{e}+03 \pm 2.34 \mathrm{e}+01$ & $6.87 \mathrm{e}+02 \pm 1.20 \mathrm{e}+02$ \\
10 & 3 & $4.67 \mathrm{e}+03 \pm 1.16 \mathrm{e}+02$ & $3.78 \mathrm{e}+03 \pm 8.06 \mathrm{e}+01$ & $1.16 \mathrm{e}+03 \pm 2.31 \mathrm{e}+02$ \\
20 & 2 & $3.37 \mathrm{e}+03 \pm 6.00 \mathrm{e}+02$ & $2.33 \mathrm{e}+03 \pm 3.67 \mathrm{e}+02$ & $1.44 \mathrm{e}+03 \pm 3.49 \mathrm{e}+02$ \\
20 & 3 & $3.60 \mathrm{e}+03 \pm 1.26 \mathrm{e}+02$ & $2.99 \mathrm{e}+03 \pm 7.19 \mathrm{e}+01$ & $1.19 \mathrm{e}+03 \pm 3.88 \mathrm{e}+02$ \\
30 & 2 & $3.17 \mathrm{e}+03 \pm 1.95 \mathrm{e}+02$ & $2.65 \mathrm{e}+03 \pm 1.39 \mathrm{e}+02$ & $1.24 \mathrm{e}+03 \pm 2.27 \mathrm{e}+02$ \\
30 & 3 & $3.82 \mathrm{e}+03 \pm 8.05 \mathrm{e}+01$ & $3.30 \mathrm{e}+03 \pm 9.12 \mathrm{e}+01$ & $1.03 \mathrm{e}+03 \pm 2.36 \mathrm{e}+02$ \\
50 & 2 & $3.48 \mathrm{e}+03 \pm 8.65 \mathrm{e}+01$ & $3.16 \mathrm{e}+03 \pm 1.28 \mathrm{e}+02$ & $6.80 \mathrm{e}+02 \pm 3.26 \mathrm{e}+01$ \\
50 & 3 & $4.12 \mathrm{e}+03 \pm 1.46 \mathrm{e}+02$ & $3.63 \mathrm{e}+03 \pm 1.15 \mathrm{e}+02$ & $7.42 \mathrm{e}+02 \pm 1.09 \mathrm{e}+02$ \\
100 & 2 & $3.72 \mathrm{e}+03 \pm 1.32 \mathrm{e}+02$ & $3.55 \mathrm{e}+03 \pm 2.12 \mathrm{e}+02$ & $1.41 \mathrm{e}+03 \pm 6.60 \mathrm{e}+02$ \\
100 & 3 & $5.07 \mathrm{e}+03 \pm 1.19 \mathrm{e}+02$ & $4.73 \mathrm{e}+03 \pm 1.79 \mathrm{e}+02$ & $1.51 \mathrm{e}+03 \pm 9.76 \mathrm{e}+01$ \\
\hline
\end{tabular}

Overall, RF-CMOCO and RF-CMOCO(FS) are two best-performing algorithms, SAEAs (RF-CMOCO, RF$\mathrm{CMOCO}(\mathrm{FS})$, and RBF-CMOCO) outperform MOEAs without the assistance of surrogates. Note however, RBF-CMOCO performs worse than NSGA-II on the 10-item MOKP instances. Table III lists the runtime of RF-CMOCO, RF$\mathrm{CMOCO}(\mathrm{FS})$, and RBF-CMOCO, from which we can see that RF-CMOCO(FS) is sped up by using feature selection. However, RF-CMOCO(FS) still requires longer time than RBF-CMOCO, which is reasonable as each RF model is an ensemble consisting of 100 CARTs and an RBF is a single model. Since the search space of the low-dimensional MOKP instances is relatively small, MOEAs are able to find the optimum with a small number of fitness evaluations. As a result, all SAEAs perform worse than the MOEAs without using surrogates probably because the approximation errors of the surrogates may slightly disturb the search. However, RBF-CMOCO outperforms NSGA-II and MOEA/D as the number of decision variables increases. Of the three SAEAs, both RF-CMOCO variants perform better than RBF-CMOCO, indicating that the RF models are advantageous over the RBF models on surrogate-assisted combinatorial optimization. Comparing RF-CMOCO with RF-CMOCO(FS), we see that they perform similarly on the MOKPs with 10-50 items, while RF-CMOCO(FS) outperforms RF-CMOCO on the 100-item MOKP instances.

To investigate the scalability of RF-CMOCO to the number of constraints, we include four additional constraints to the biobjective MOKP instance with 20 items. Note that for a fair comparison, the feasible region of each additional constraint is set to cover the PS of the original MOKP instance to keep the PF unchanged. The IGD values obtained by the RFCMOCO on the MOKP with different constraints over 30 runs are shown in Fig. 8. The performance of RF-CMOCO decreases when the number of constraints increases due to the accumulated error of surrogate models. However, the performance does not significantly deteriorate when the number of constraints is larger than three. Therefore, the scalability of RF-CMOCO to the number of constraints is acceptable.

We can draw three conclusions from the above experimental results. Firstly, surrogate models can effectively save the computation cost in solving data-driven constrained multiobjective combinatorial optimization problems. Secondly, the RF models are better suited for combinatorial optimization problems than the RBF models. Thirdly, the performance of the RF models degenerates on high-dimensional combinatorial optimization problems, which can be mitigated to a certain 


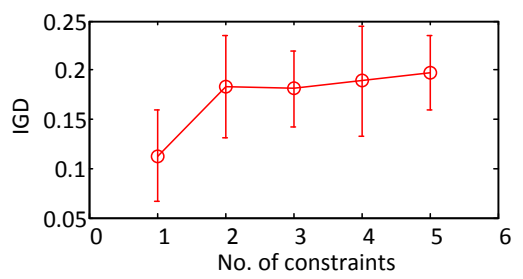

Fig. 8. IGD values obtained by RF-CMOCO on the bi-objective MOKP instance with 20 items when the number of constraints increases.

extent using dimension reduction techniques.

\section{Design of Trauma Systems}

In this section, we apply the proposed algorithms to the design of two trauma systems, one in Scotland and the other in Colorado in the US. Trauma system design can be formulated as a bi-objective constrained combinatorial optimization problem [5] and the details of the problem formulation will be discussed below.

\section{A. Design of the Scotland Trauma System}

In Scotland, 18 existing hospitals can be classified into three different categories: major trauma centres (MTC), trauma units (TU), and local emergency hospitals (LEH). The decision variables of the trauma system design problem are the categories of the 18 hospitals. Different configurations (categories of the hospitals) lead to different clinic and resource outcomes, which are hard to be analytically evaluated. To address this issue, 40,000 emergency incidents within one year are used to design the trauma system, which is a typical data-driven optimization problem [5].

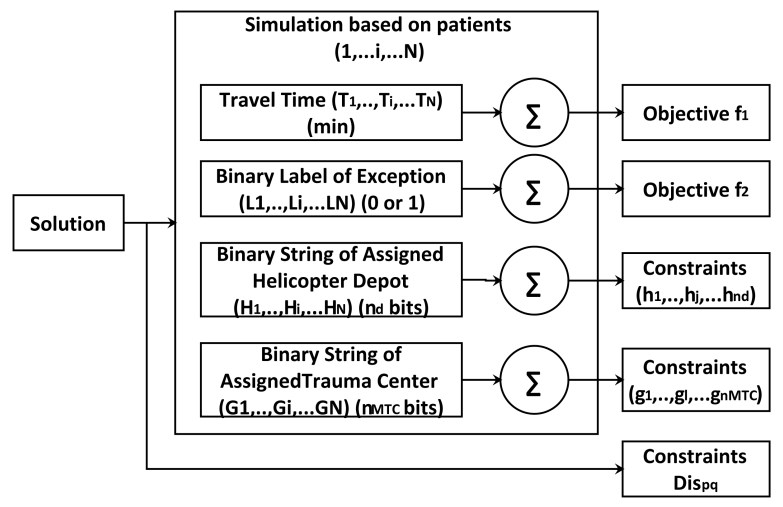

Fig. 9. Data-driven evaluation of the objectives and constraints in trauma system design.

The total transportation time for the patients recorded in the data and the number of MTC exceptions are two objectives $\left(f_{1}\right.$ and $\left.f_{2}\right)$ to be minimized. By an MTC exception, we mean a case where a patient who should be triaged to an MTC has to be sent to a TU since there is no MTC near the accident location. A binary bit $L_{i}$ is set 1 if patient $i$ is an MTC exception, otherwise it is set as 0 . In addition, three constraints need to be considered, namely, the number of helicopters used for transporting patients in depot $j$ should not exceed its maximum number $n_{h}^{j}$; the number of patients handled by each MTC per day should be larger than a predefined threshold $V$; and the distance between any two TUs should be larger than $d_{T U}$. The two objectives $\left(f_{1}\right.$ and $\left.f_{2}\right)$ and the three constraints are defined as follows:

$$
\begin{gathered}
\min \left(f_{1}=\sum_{i=1}^{N} T_{i}, f_{2}=\sum_{i=1}^{N} L_{i}\right) \\
h_{j}=\sum_{i=1}^{N} H_{i}^{j} \leq D n_{h}^{j}, 1 \leq j \leq n_{d} \\
g_{l}=\sum_{i=1}^{N} G_{i}^{l} \geq D V, 1 \leq l \leq n_{M T C} . \\
D i s_{p q} \geq d_{T U}, 1 \leq p \leq n_{T u}, 1 \leq q \leq n_{T u}, p \neq q
\end{gathered}
$$

Note that the two objectives as well as the first two constraints are calculated using the 40,000 data records, while the third constraint can be calculated straightforwardly based on the location of the TUs. Because of the large number of records, the evaluation for a single configuration is computationally expensive. The main steps for calculating the objectives and constraints are given in Fig. 9.

The details of the patient allocation algorithm can be found in [5], [83]. Given a patient $i$ and a candidate configuration with $n_{M T C}$ MTCs and $n_{T U}$ TUs, a nearby and appropriate hospital center is assigned, the transportation time $T_{i}$ is the time for transporting the $i$-th patient from the accident location to the assigned hospital. If a helicopter is used for transporting the $i$ patient from the $j$-th $\left(1 \leq j \leq n_{d}\right)$ depot, the $j$-th bit of a binary string $H_{i}$ is set to 1 , otherwise all $n_{d}$ bits of $H_{i}$ are set to 0 . If the assigned hospital is the $l$-th MTC $\left(1 \leq l \leq n_{M T C}\right.$ in the solution, the $l$-th bit of a binary string $G_{i}$ is set to 1 , otherwise all $n_{M T C}$ bits of $G_{i}$ are set to 0 .

We compare the performance of RF-CMOCO, RFCMOCO(FS), RBF-CMOCO, and NSGA-II for solving the Scottish trauma system design problem (MOEA/D is not included here due to its poor performance on the benchmark problems). Each compared algorithm is run for 20 times. The settings of the algorithms are presented in Table I. Similarly, we use IGD to evaluate the performance of RF-CMOCO and NSGA-II, where the reference set is generated as in [5]. The population size of NSGA-II is set to 100 and the algorithm is run for 200 generations using exact function evaluations, meaning that the objectives and first two constraints are calculated using all 40,000 data records. Each compared algorithm is repeated for five runs. Then the non-dominated solutions of the non-dominated solutions obtained from the five runs are used as the reference set for calculating the IGD. The objectives are also normalized using the extreme points in the reference set.

The IGD values of RF-CMOCO, RF-CMOCO(FS), RBFCMOCO and NSGA-II are given in Table IV. Using only 1500 exact function evaluations, RF-CMOCO, RF-CMOCO(FS) and RBF-CMOCO are able to obtain solutions closer to the reference set obtained by NSGA-II, and RF-CMOCO achieves the best IGD value. As the solutions obtained by NSGA-II are all infeasible, Fig. 10 shows the non-dominated solution 

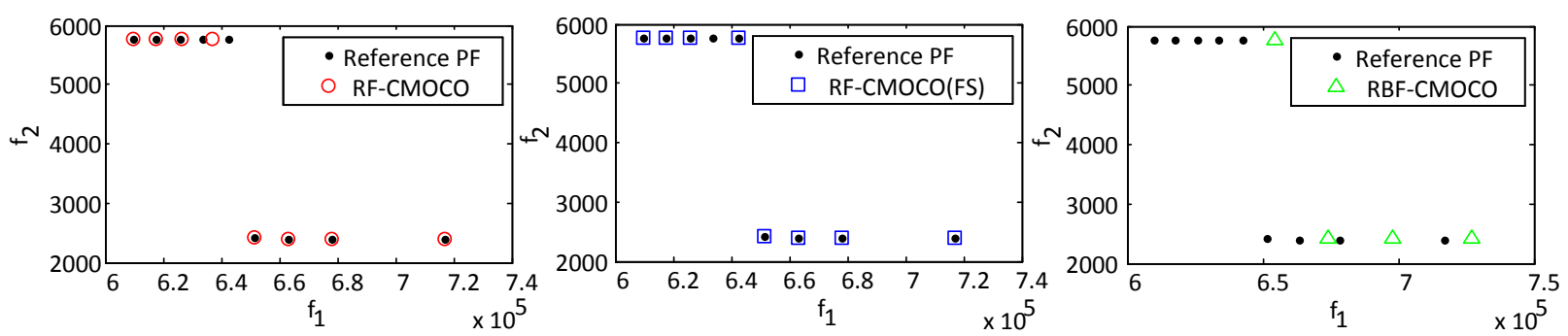

Fig. 10. Non-dominated solution set of the Scotland trauma system design problem obtained by RF-CMOCO, RF-CMOCO(FS), and RBF-CMOCO.

set obtained by RF-CMOCO, RF-CMOCO(FS) and RBFCMOCO with the median IGD value only.

TABLE IV

THE IGD VALUES OF RF-CMOCO, RF-CMOCO(FS), RBF-CMOCO AND NSGA-II OBTAINED ON THE SCOTLAND TRAUMA SYSTEM DESIGN PROBLEM. THE RESULTS OF THE BEST AND SECOND BEST ALGORITHMS FOR EACH PROBLEM ARE HIGHLIGHTED IN GRAY AND LIGHT GRAY.

\begin{tabular}{cl}
\hline RF-CMOCO & $2.02 \mathrm{e}-01 \pm 2.75 \mathrm{e}-01$ \\
RF-CMOCO(FS) & $2.71 \mathrm{e}-01 \pm 2.76 \mathrm{e}-01$ \\
RBF-CMOCO & $3.48 \mathrm{e}-01 \pm 2.48 \mathrm{e}-01$ \\
NSGA-II & $1.56 \mathrm{e}+00 \pm 3.81 \mathrm{e}-01$ \\
\hline
\end{tabular}

The results demonstrate that surrogate-assisted MOEAs outperform NSGA-II. Among the three surrogate-assisted algorithms, RF-CMOCO has achieved better convergence performance than RBF-CMOCO, indicating the RF model is better suited for combinatorial optimization problems than the RBF model. Similar to the findings in Section III-A, RF$\mathrm{CMOCO}(\mathrm{FS})$ performs worse than RF-CMOCO on this 18dimensional trauma system design problem.

\section{B. Colorado Trauma System Design}

In this subsection, we apply the proposed algorithm to the Colorado trauma system design [84]. Different from the Scotland trauma system, the Colorado trauma system has 72 hospitals, which are categorized into five different capability levels (denoted as L1-L5). Similarly, the design of the Colorado trauma system can be formulated as a combinatorial optimization problem and the differences lie in the calculation of the number of exceptions and the MTC case volume, where MTC is changed to L1 in Eq. (8) and MTC is changed to L2 in Eq. (10), and the TU in the third constraint (TU distances in Eq. (11)) is changed to L3. Here, 100,000 emergency records in five years are available to calculate the objectives and constraints. It should be noted that the search space of the Colorado trauma system design problem becomes much larger than that of the Scotland trauma system design, and the evaluations of the objectives and constraints are more timeconsuming.

We run RF-CMOCO, RF-CMOCO(FS), RBF-CMOCO, and NSGA-II on the Colorado trauma system design problem for 20 times. Since this problem has a large search space, we change the stopping criterion from 1500 exact function evaluations to 2000 while all the rest settings remain the same as in Section III-C. The performance of the compared algorithms is evaluated in terms of IGD, where the reference PF set is the non-dominated solution set of the five solution sets obtained by running NSGA-II (population size is set to 100) for 200 generations, all using exact function evaluations. The objectives are also normalized by the extreme points in the reference set. The IGD values of RF-CMOCO, RF-CMOCO(FS), RBF-CMOCO, and NSGA-II on the Colorado trauma system design problem are listed in Table V. Given only 1500 exact function evaluations, none of the algorithms are able to obtain satisfactory results. When 2000 exact function evaluations are allowed, the performance of the compared algorithms is considerably improved except for NSGA-II. Therefore, we only present the non-dominated solution sets obtained by RFCMOCO, RF-CMOCO(FS), and RBF-CMOCO in Fig. 11 for the run having the median IGD value.

The results on the Colorado trauma system design problem are different from those on the Scotland trauma system design problem. Among the compared algorithms, RF-CMOCO(FS) performs the best in terms of the IGD value. This agrees with the findings in the benchmark problems that feature selection can help improve the performance of the proposed algorithms on high-dimensional systems, as the Colorado trauma system design problem has 72 decision variables. In addition, there are five possible capability levels for each decision variable, significantly increasing the search space compared to the Scotland trauma system design problem. The performance deterioration of the RBF-CMOCO indicates that RF models are better suited than RBF models for high-dimensional combinatorial optimization problems.

TABLE V

THE IGD VALUES OF RF-CMOCO, RF-CMOCO(FS), RBF-CMOCO, AND NSGA-II (STOPPED AFTER 1500 OR 2000 EXACT FUNCTION EVALUATIONS ARE EXHAUSTED) ON THE COLORADO TRAUMA SYSTEM DESIGN PROBLEM. THE BEST AND SECOND BEST FITNESS VALUES AMONG ALL THE COMPARED ALGORITHMS FOR EACH PROBLEM ARE HIGHLIGHTED IN GRAY AND LIGHT GRAY.

\begin{tabular}{ccc}
\hline No. of function evaluations & 2000 & 1500 \\
\hline RF-CMOCO & $2.53 \mathrm{e}+00 \pm 8.86 \mathrm{e}-01$ & $4.62 \mathrm{e}+00 \pm 7.72 \mathrm{e}-01$ \\
RF-CMOCO(FS) & $1.65 \mathrm{e}+00 \pm 1.04 \mathrm{e}-00$ & $4.20 \mathrm{e}+00 \pm 8.54 \mathrm{e}-01$ \\
RBF-CMOCO & $2.96 \mathrm{e}+00 \pm 6.90 \mathrm{e}-01$ & $7.23 \mathrm{e}+00 \pm 1.66 \mathrm{e}-00$ \\
NSGA-II & $4.50 \mathrm{e}+00 \pm 1.48 \mathrm{e}+00$ & $7.54 \mathrm{e}+00 \pm 2.01 \mathrm{e}-00$ \\
\hline
\end{tabular}

As shown in Fig. 11, the solution set obtained by RF$\mathrm{CMOCO}(\mathrm{FS})$ is still much worse than the reference PF. Note that the computation time consumed for obtaining the reference $\mathrm{PF}$ and that for the compared algorithms that use a maximum of 2000 exact function evaluations are different, as detailed in Table VI. It takes about 56 hours for the NSGA-II to 


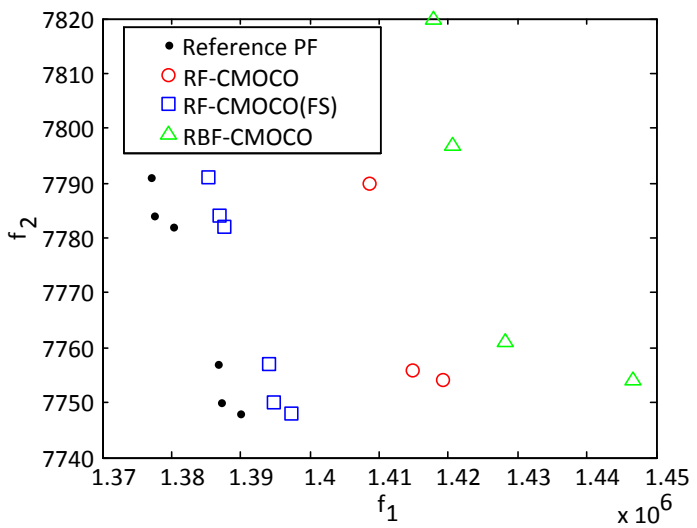

Fig. 11. Non-dominated solution set of the Colorado trauma system design problem obtained by RF-CMOCO, RF-CMOCO(FS), and RBF-CMOCO using 2000 exact function evaluations.

obtain the reference PF. By contrast, the compared algorithms consume only about 3 hours to obtain the results. Therefore, RF-CMOCO(FS) is still very competitive taking into account the fact that it consumes much less computation time than NSGA-II.

TABLE VI

THE AVERAGE RUNTIME (H) OF NSGA-II (FOR OBTAINING THE REFERENCE PF), RF-CMOCO, RF-CMOCO(FS), RBF-CMOCO, AND

NSGA-II (USING A MAXIMUM OF 2000 EXACT FUNCTION EVALUATIONS) ON COLORADO TRAUMA SYSTEM DESIGN PROBLEM

\begin{tabular}{cc}
\hline NSGA-II for reference PF & 55.85 \\
RF-CMOCO & 3.21 \\
RF-CMOCO(FS) & 3.36 \\
RBF-CMOCO & 3.34 \\
NSGA-II & 2.73 \\
\hline
\end{tabular}

From the two different trauma system design problems, we can conclude that RF-CMOCO is effective for solving expensive data-driven combinatorial optimization problems. Based on the comparative results, we can also conclude that the RF models are better suited as surrogates for combinatorial optimization problems than the RBF models. Finally, the results demonstrate that dimension reduction techniques such as feature selection are able to enhance the performance of RF-assisted EAs for solving high-dimensional combinatorial optimization problems.

\section{CONCluding Remarks}

In this paper, we present a random forest assisted evolutionary algorithm for solving expensive data-driven constrained multi-objective combinatorial optimization problems. We show that random forest models work better than radialbasis-function network models as surrogates for combinatorial optimization problems. To address the errors introduced by the surrogates for the constraints, we design a correction boundary strategy with the help of logistic regression models and a stochastic ranking strategy using two selection criteria, one considering the problem as an unconstrained multi-objective problem and the other as a constrained multi-objective problem. Our empirical results demonstrate that the proposed constraint handling strategies based on logistic regression correction and stochastic ranking can improve the selection accuracy up to $70 \%-80 \%$ in the optimization process. The effectiveness of the RF-CMOCO has been verified on both multi-objective knapsack problems and two real-world datadriven trauma system design problems.

Although the effectiveness and efficiency of the proposed algorithms are promising in that they can significantly reduce the computation time at the price of slight quality degradation, some open issues remain to be resolved in the future. First, there is much room for improving the performance of random forest models, and better surrogate models for approximating the objective and constraint functions are in high demand. Second, indirect surrogate models for permutation or tree structures need to be studied so that surrogate-assisted evolutionary algorithms can be applied to a wider range of combinatorial optimization problems. Finally, more sophisticated machine learning techniques are required to determine whether a solution is feasible or not, which heavily influences the performance of surrogate-assisted evolutionary optimization of expensive constrained problems.

\section{ACKNOWLEDGEMENT}

The authors would like to thank Dr Jan O Jansen, Dr Ernest E Moore, Dr Jonathan J Morrison, Dr James D Hutchison, and Dr Marion K Campbell for providing the data of the Colorado trauma system problem.

\section{REFERENCES}

[1] K. L. Hoffman, "Combinatorial optimization: Current successes and directions for the future," Journal of computational and applied mathematics, vol. 124, no. 1, pp. 341-360, 2000.

[2] M. Ehrgott and X. Gandibleux, "A survey and annotated bibliography of multiobjective combinatorial optimization," OR Spectrum, vol. 22, no. 4, pp. 425-460, 2000.

[3] K. Florios, G. Mavrotas, and D. Diakoulaki, "Solving multiobjective, multiconstraint knapsack problems using mathematical programming and evolutionary algorithms," European Journal of Operational Research, vol. 203, no. 1, pp. 14-21, 2010.

[4] Y. Jin, "A comprehensive survey of fitness approximation in evolutionary computation," Soft Computing, vol. 9, no. 1, pp. 3-12, 2005.

[5] H. Wang, Y. Jin, and J. O. Janson, "Data-driven surrogate-assisted multi-objective evolutionary optimization of a trauma system," IEEE Transactions on Evolutionary Computation, vol. 20, no. 6, pp. 939-952, 2016.

[6] Y. Jin, "Surrogate-assisted evolutionary computation: Recent advances and future challenges," Swarm and Evolutionary Computation, vol. 1, no. 2, pp. 61-70, 2011

[7] Y. Jin and B. Sendhoff, "A systems approach to evolutionary multiobjective structural optimization and beyond," IEEE Computational Intelligence Magazine, vol. 4, no. 3, pp. 62-76, 2009.

[8] H. Wang, J. Doherty, and Y. Jin, "Hierarchical surrogate-assisted evolutionary multi-scenario airfoil shape optimization," in Proceedings of the 2018 IEEE World Congress on Computational Intelligence (WCCI 2018). IEEE, 2018.

[9] S. Koziel and A. Bekasiewicz, "Rapid simulation-driven multiobjective design optimization of decomposable compact microwave passives," IEEE Transactions on Microwave Theory and Techniques, vol. 64, no. 8, pp. 2454-2461, 2016.

[10] D. Guo, T. Chai, J. Ding, and Y. Jin, "Small data driven evolutionary multi-objective optimization of fused magnesium furnaces," in IEEE Symposium Series on Computational Intelligence. Athens, Greece: IEEE, December 2016.

[11] B. Liu, Q. Zhang, and G. G. Gielen, "A Gaussian process surrogate model assisted evolutionary algorithm for medium scale expensive optimization problems," IEEE Transactions on Evolutionary Computation, vol. 18, no. 2, pp. 180-192, 2014. 
[12] R. Allmendinger, M. T. M. Emmerich, J. Hakanen, Y. Jin, and E. Rigoni, "Surrogate-assisted multicriteria optimization: Complexities, prospective solutions, and business case," Journal of Multi-Criteria Decision Analysis, vol. 14, pp. 5-25, 2017.

[13] T. Chugh, Y. Jin, K. Miettinen, J. Hakanen, and K. Sindhya, "A surrogate-assisted reference vector guided evolutionary algorithm for computationally expensive many-objective optimization," IEEE Transactions on Evolutionary Computation, vol. 22, no. 1, pp. 129-142, 2018

[14] L. Willmes, T. Back, Y. Jin, and B. Sendhoff, "Comparing neural networks and kriging for fitness approximation in evolutionary optimization," in IEEE Congress on Evolutionary Computation, vol. 1. IEEE, 2003, pp. 663-670.

[15] R. G. Regis, "Evolutionary programming for high-dimensional constrained expensive black-box optimization using radial basis functions," IEEE Transactions on Evolutionary Computation, vol. 18, no. 3, pp. 326-347, 2014.

[16] C. Sun, Y. Jin, J. Zeng, and Y. Yu, "A two-layer surrogate-assisted particle swarm optimization algorithm," Soft Computing, vol. 19, no. 6, pp. 1461-1475, 2015.

[17] Z. Zhou, Y. S. Ong, P. B. Nair, A. J. Keane, and K. Y. Lum, "Combining global and local surrogate models to accelerate evolutionary optimization," IEEE Transactions on Systems, Man, and Cybernetics, Part C: Applications and Reviews, vol. 37, no. 1, pp. 66-76, 2007.

[18] H. Wang, Y. Jin, C. Sun, and J. Doherty, "Offline datadriven evolutionary optimization using selective surrogate ensembles," IEEE Transactions on Evolutionary Computation, 2018, accepted, DOI=0.1109/TEVC.2018.2834881.

[19] Z. Zhou, Y. S. Ong, M. H. Nguyen, and D. Lim, "A study on polynomial regression and Gaussian process global surrogate model in hierarchical surrogate-assisted evolutionary algorithm," in IEEE Congress on Evolutionary Computation, vol. 3. IEEE, 2005, pp. 2832-2839.

[20] Y. Jin, M. Olhofer, and B. Sendhoff, "A framework for evolutionary optimization with approximate fitness functions," IEEE Transactions on Evolutionary Computation, vol. 6, no. 5, pp. 481-494, 2002.

[21] M. Hüsken, Y. Jin, and B. Sendhoff, "Structure optimization of neural networks for evolutionary design optimization," Soft Computing, vol. 9 , no. 1, pp. 21-28, 2005.

[22] D. Lim, Y. Jin, Y.-S. Ong, and B. Sendhoff, "Generalizing surrogateassisted evolutionary computation," IEEE Transactions on Evolutionary Computation, vol. 14, no. 3, pp. 329-355, 2010.

[23] A. Husain and K.-Y. Kim, "Enhanced multi-objective optimization of a microchannel heat sink through evolutionary algorithm coupled with multiple surrogate models," Applied Thermal Engineering, vol. 30 no. 13, pp. 1683-1691, 2010.

[24] D. Lim, Y.-S. Ong, Y. Jin, and B. Sendhoff, "A study on metamodeling techniques, ensembles, and multi-surrogates in evolutionary computation," in Proceedings of the 9th Annual Conference on Genetic and Evolutionary Computation. ACM, 2007, pp. 1288-1295.

[25] H. Wang, Y. Jin, and J. Doherty, "Committee-based active learning for surrogate-assisted particle swarm optimization of expensive problems," IEEE Transactions on Cybernetics, vol. 47, no. 9, pp. 2664-2677, 2017.

[26] Y. Jin, M. Olhofer, and B. Sendhoff, "On evolutionary optimization with approximate fitness functions," in Proceedings of the 2nd Annual Conference on Genetic and Evolutionary Computation. Morgan Kaufmann Publishers Inc., 2000, pp. 786-793.

[27] J. Branke and C. Schmidt, "Faster convergence by means of fitness estimation," Soft Computing, vol. 9, no. 1, pp. 13-20, 2005.

[28] M. T. Emmerich, K. C. Giannakoglou, and B. Naujoks, "Single-and multiobjective evolutionary optimization assisted by gaussian random field metamodels," IEEE Transactions on Evolutionary Computation, vol. 10, no. 4, pp. 421-439, 2006.

[29] Y. S. Ong, P. B. Nair, and A. J. Keane, "Evolutionary optimization of computationally expensive problems via surrogate modeling," AIAA journal, vol. 41, no. 4, pp. 687-696, 2003.

[30] C. Sun, Y. Jin, R. Cheng, J. Ding, and J. Zeng, "Surrogate-assisted cooperative swarm optimization of high-dimensional expensive problems," IEEE Transactions on Evolutionary Computation, vol. 21, no. 4, pp. 644-660, 2017.

[31] J. Knowles, "ParEGO: a hybrid algorithm with on-line landscape approximation for expensive multiobjective optimization problems," IEEE Transactions on Evolutionary Computation, vol. 10, no. 1, pp. 50-66, 2006.

[32] Q. Zhang, W. Liu, E. Tsang, and B. Virginas, "Expensive multiobjective optimization by MOEA/D with gaussian process model," IEEE Transactions on Evolutionary Computation, vol. 14, no. 3, pp. 456-474, 2010.
[33] L. Pan, C. He, Y. Tian, H. Wang, X. Zhang, and Y. Jin, "A classification based surrogate-assisted evolutionary algorithm for expensive manyobjective optimization," IEEE Transactions on Evolutionary Computation, 2018, accepted, DOI=10.1109/TEVC.2018.2802784.

[34] T. Bartz-Beielstein and M. Zaefferer, "Model-based methods for continuous and discrete global optimization," Applied Soft Computing, vol. 55, pp. 154-167, 2017.

[35] F. Hutter, H. H. Hoos, and K. Leyton-Brown, "Sequential model-based optimization for general algorithm configuration." LION, vol. 5, pp. 507523, 2011.

[36] R. Li, M. Emmerich, J. Eggermont, and E. Bovenkamp, "Mixedinteger optimization of coronary vessel image analysis using evolution strategies," in Proceedings of the 8th Annual Conference on Genetic and Evolutionary Computation, 2006, pp. 1645-1652.

[37] L. Zhuang, K. Tang, and Y. Jin, "Metamodel assisted mixed-integer evolution strategies based on kendall rank correlation coefficient," in International Conference on Intelligent Data Engineering and Automated Learning. Springer, 2013, pp. 366-375.

[38] A. Moraglio and A. Kattan, "Geometric generalisation of surrogate model based optimisation to combinatorial spaces," in European Conference on Evolutionary Computation in Combinatorial Optimization. Springer, 2011, pp. 142-154.

[39] R. van der Merwe, T. K. Leen, Z. Lu, S. Frolov, and A. M. Baptista, "Fast neural network surrogates for very high dimensional physics-based models in computational oceanography," Neural Networks, vol. 20, no. 4 pp. 462-478, 2007.

[40] D.-J. Wang, F. Liu, Y.-Z. Wang, and Y. Jin, "A knowledge-based evolutionary proactive scheduling approach in the presence of machine breakdown and deterioration effect," Knowledge-Based Systems, vol. 90, pp. 70-80, 2015.

[41] S. Nguyen, M. Zhang, and K. C. Tan, "Surrogate-assisted genetic programming with simplified models for automated design of dispatching rules," IEEE transactions on cybernetics, vol. 47, no. 9, pp. 2951-2965, 2017.

[42] I. Voutchkov, A. Keane, A. Bhaskar, and T. M. Olsen, "Weld sequence optimization: the use of surrogate models for solving sequential combinatorial problems," Computer methods in applied mechanics and engineering, vol. 194, no. 30, pp. 3535-3551, 2005.

[43] B. Yuan, B. Li, T. Weise, and X. Yao, "A new memetic algorithm with fitness approximation for the defect-tolerant logic mapping in crossbar-based nanoarchitectures," IEEE Transactions on Evolutionary Computation, vol. 18, no. 6, pp. 846-859, 2014.

[44] Z. Cai and Y. Wang, "A multiobjective optimization-based evolutionary algorithm for constrained optimization," IEEE Transactions on Evolutionary Computation, vol. 10, no. 6, pp. 658-675, 2006.

[45] M. Kazemi, G. G. Wang, S. Rahnamayan, and K. Gupta, "Metamodelbased optimization for problems with expensive objective and constraint functions," Journal of Mechanical Design, vol. 133, no. 1, p. 014505 , 2011.

[46] K. S. Won and T. Ray, "A framework for design optimization using surrogates," Engineering Optimization, vol. 37, no. 7, pp. 685-703, 2005.

[47] C. A. C. Coello, "Theoretical and numerical constraint-handling techniques used with evolutionary algorithms: a survey of the state of the art," Computer methods in applied mechanics and engineering, vol. 191, no. 11 , pp. 1245-1287, 2002.

[48] H. K. Singh, T. Ray, and W. Smith, "Surrogate assisted simulated annealing (SASA) for constrained multi-objective optimization," in IEEE Congress on Evolutionary Computation. IEEE, 2010, pp. 1-8.

[49] J. M. Parr, A. I. Forrester, A. J. Keane, and C. M. Holden, "Enhancing infill sampling criteria for surrogate-based constrained optimization," Journal of Computational Methods in Sciences and Engineering, vol. 12, no. 1, 2, pp. 25-45, 2012.

[50] J. Poloczek and O. Kramer, "Local SVM constraint surrogate models for self-adaptive evolution strategies," in Annual Conference on Artificial Intelligence. Springer, 2013, pp. 164-175.

[51] S. D. Handoko, C. K. Kwoh, and Y.-S. Ong, "Feasibility structure modeling: an effective chaperone for constrained memetic algorithms," IEEE Transactions on Evolutionary Computation, vol. 14, no. 5, pp. $740-758,2010$.

[52] Y. Tenne, K. Izui, and S. Nishiwaki, "Handling undefined vectors in expensive optimization problems," in European Conference on the Applications of Evolutionary Computation. Springer, 2010, pp. 582591.

[53] O. Kramer, A. Barthelmes, and G. Rudolph, "Surrogate constraint functions for CMA evolution strategies," in Annual Conference on Artificial Intelligence. Springer, 2009, pp. 169-176. 
[54] Y. Jin, S. Oh, and M. Jeon, "Incremental approximation of nonlinear constraint functions for evolutionary constrained optimization," in IEEE Congress on Evolutionary Computation. IEEE, 2010, pp. 1-8.

[55] T. Chugh, K. Sindhya, K. Miettinen, J. Hakanen, and Y. Jin, "On constraint handling in surrogate-assisted evolutionary many-objective optimization," in International Conference on Parallel Problem Solving from Nature. Springer, 2016, pp. 214-224.

[56] M. Pilát and R. Neruda, "Feature extraction for surrogate models in genetic programming," in International Conference on Parallel Problem Solving from Nature. Springer, 2016, pp. 335-344.

[57] T. P. Runarsson and X. Yao, "Stochastic ranking for constrained evolutionary optimization," IEEE Transactions on Evolutionary Computation, vol. 4, no. 3, pp. 284-294, 2000.

[58] K. Miettinen, Nonlinear multiobjective optimization. Springer Science \& Business Media, 2012, vol. 12.

[59] A. Zhou, B.-Y. Qu, H. Li, S.-Z. Zhao, P. N. Suganthan, and Q. Zhang, "Multiobjective evolutionary algorithms: A survey of the state of the art," Swarm and Evolutionary Computation, vol. 1, no. 1, pp. 32-49, 2011.

[60] K. Deb, A. Pratap, S. Agarwal, and T. Meyarivan, "A fast and elitist multiobjective genetic algorithm: NSGA-II," IEEE Transactions on Evolutionary Computation, vol. 6, no. 2, pp. 182-197, 2002.

[61] Q. Zhang and H. Li, "MOEA/D: A multiobjective evolutionary algorithm based on decomposition," IEEE Transactions on Evolutionary Computation, vol. 11, no. 6, pp. 712-731, 2007.

[62] J. Bader and E. Zitzler, "HypE: an algorithm for fast hypervolume-based many-objective optimization," Evolutionary Computation, vol. 19, no. 1, pp. 45-76, 2011.

[63] A. Liaw and M. Wiener, "Classification and regression by random forest," $R$ news, vol. 2, no. 3, pp. 18-22, 2002.

[64] D. Steinberg and P. Colla, "CART: classification and regression trees," The Top Ten Algorithms in Data Mining, vol. 9, p. 179, 2009.

[65] L. Breiman, "Random forests," Machine Learning, vol. 45, no. 1, pp. $5-32,2001$

[66] A. Verikas, A. Gelzinis, and M. Bacauskiene, "Mining data with random forests: A survey and results of new tests," Pattern Recognition, vol. 44 no. 2, pp. 330-349, 2011.

[67] L. Breiman, J. Friedman, C. J. Stone, and R. A. Olshen, Classification and regression trees. CRC press, 1984.

[68] M. Kendall and J. D. Gibbons, Rank Correlation Methods. Edward Arnold, 1990

[69] H. Wang and X. Yao, "Corner sort for pareto-based many-objective optimization," IEEE Transactions on Cybernetics, vol. 44, no. 1, pp. 92-102, 2014.

[70] D. A. Freedman, Statistical models: theory and practice. cambridge university press, 2009.

[71] J. Parr, A. Keane, A. I. Forrester, and C. Holden, "Infill sampling criteria for surrogate-based optimization with constraint handling," Engineering Optimization, vol. 44, no. 10, pp. 1147-1166, 2012.

[72] C. K. Goh, D. Lim, L. Ma, Y.-S. Ong, and P. S. Dutta, "A surrogateassisted memetic co-evolutionary algorithm for expensive constrained optimization problems," in IEEE Congress on Evolutionary Computation. IEEE, 2011, pp. 744-749.

[73] Y. Tian, H. Wang, X. Zhang, and Y. Jin, "Effectiveness and efficiency of non-dominated sorting for evolutionary multi- and many-objective optimization," Complex \& Intelligent Systems, vol. 3, no. 4, pp. 247263, 2017

[74] S. Boriah, V. Chandola, and V. Kumar, "Similarity measures for categorical data: A comparative evaluation," in Proceedings of the 2008 SIAM International Conference on Data Mining. SIAM, 2008, pp. 243-254.

[75] E. L. Ulungu and J. Teghem, "Multi-objective combinatorial optimization problems: A survey," Journal of Multi-Criteria Decision Analysis, vol. 3, no. 2, pp. 83-104, 1994

[76] G. Kirlik and S. Sayın, "A new algorithm for generating all nondominated solutions of multiobjective discrete optimization problems," European Journal of Operational Research, vol. 232, no. 3, pp. 479-488, 2014.

[77] C. Bazgan, H. Hugot, and D. Vanderpooten, "Solving efficiently the 0-1 multi-objective knapsack problem," Computers \& Operations Research, vol. 36, no. 1, pp. 260-279, 2009.

[78] T. Lust and J. Teghem, "The multiobjective multidimensional knapsack problem: a survey and a new approach," International Transactions in Operational Research, vol. 19, no. 4, pp. 495-520, 2012.

[79] A. Duarte, J. J. Pantrigo, E. G. Pardo, and N. Mladenovic, "Multiobjective variable neighborhood search: an application to combinatorial optimization problems," Journal of Global Optimization, vol. 63, no. 3, pp. 515-536, 2015 .
[80] Q. Zhang, A. Zhou, S. Zhao, P. Suganthan, W. Liu, and S. Tiwari, "Multiobjective optimization test instances for the CEC 2009 special session and competition," University of Essex, Colchester, UK and Nanyang Technological University, Singapore, Special Session on Performance Assessment of Multi-Objective Optimization Algorithms, Technical Report, Tech. Rep., 2008.

[81] M. Hollander and D. Wolfe, Nonparametric statistical methods. WileyInterscience, 1999.

[82] D. Hadka and P. Reed, "Diagnostic assessment of search controls and failure modes in many-objective evolutionary optimization," Evolutionary Computation, vol. 20, no. 3, pp. 423-452, 2012.

[83] J. O. Jansen and M. K. Campbell, "The GEOS study: Designing a geospatially optimised trauma system for scotland," The Surgeon, vol. 12, no. 2, pp. 61-63, 2014.

[84] J. Jansen, E. Moore, H. Wang, J. Morrison, J. Hutchison, M. Campbell, and A. Sauaia, "Maximizing geographical efficiency: An analysis of the configuration of colorado's trauma system." The journal of trauma and acute care surgery, vol. 84, no. 5, pp. 762-770, 2018

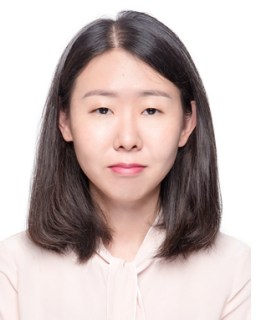

Handing Wang (S'10-M'16) received the B.Eng. and Ph.D. degrees from Xidian University, Xi'an, China, in 2010 and 2015, respectively.

She is currently a professor with School of Artificial Intelligence, Xidian University, Xi'an, China.

Dr. Wang is an Associate Editor of IEEE Computational Intelligence Magazine and Complex \& Intelligent Systems, chair of the Task Force on Intelligence Systems for Health within the Intelligent Systems Applications Technical Committee of IEEE Computational Intelligence Society. Her research interests include nature-inspired computation, multiobjective optimization, multiple criteria decision making, surrogate-assisted evolutionary optimization, and real-world problems.

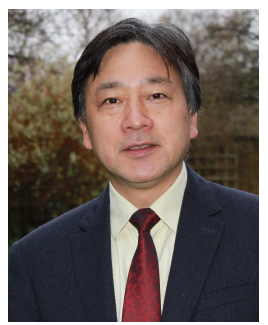

Yaochu Jin (M'98-SM'02-F'16) received the B.Sc., M.Sc., and Ph.D. degrees from Zhejiang University, Hangzhou, China, in 1988, 1991, and 1996 respectively, and the Dr.-Ing. degree from Ruhr University Bochum, Germany, in 2001.

$\mathrm{He}$ is a Professor in Computational Intelligence, Department of Computer Science, University of Surrey, Guildford, U.K., where he heads the Nature Inspired Computing and Engineering Group. He is also a Finland Distinguished Professor funded by the Finnish Funding Agency for Innovation (Tekes) and a Changjiang Distinguished Visiting Professor appointed by the Ministry of Education, China. His research interests lie primarily in the crossdisciplinary areas of computational intelligence, computational neuroscience, and computational systems biology. He is also particularly interested in the application of nature-inspired algorithms to solving real-world optimization, learning and self-organization problems. He has (co)authored over 300 peerreviewed journal and conference papers and been granted eight patents on evolutionary optimization.

Dr Jin is the Editor-in-Chief of the IEEE TRANSACTIONS ON COGNITIVE AND DEVELOPMENTAL SYSTEMS and Complex \& Intelligent Systems. He is an IEEE Distinguished Lecturer (2017-2019) and was the Vice President for Technical Activities of the IEEE Computational Intelligence Society (2014-2015). He is a recipient of the 2014 and 2016 IEEE Computational Intelligence Magazine Outstanding Paper Award, and the 2017 IEEE Transactions on Evolutionary Computation Outstanding Paper Award. He is a Fellow of IEEE. 